\title{
DISTRIBUTION FUNCTIONS OF RATIO SEQUENCES. AN EXPOSITORY PAPER
}

\author{
Oto Strauch
}

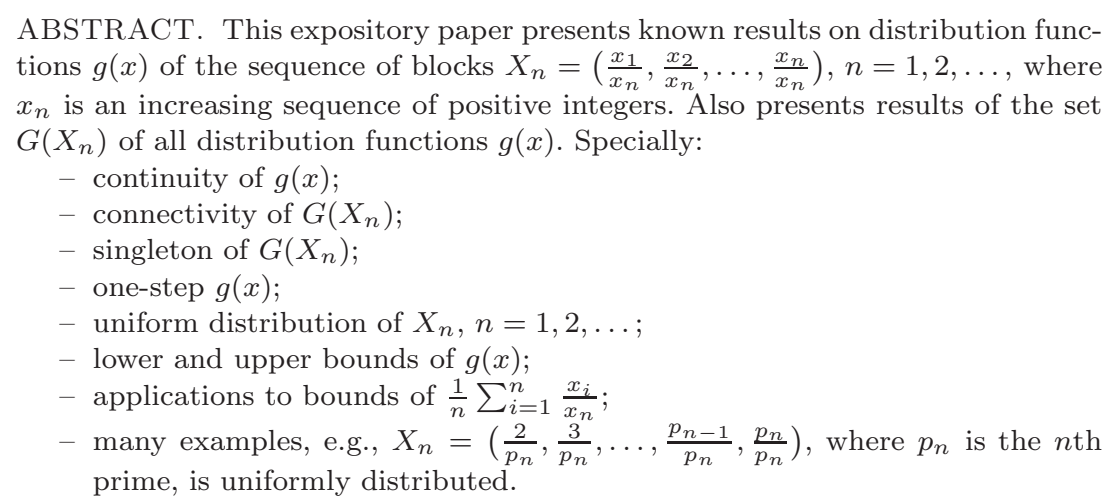

The present results have been published by 25 papers of several authors between 2001-2013.

\section{Introduction}

Let $x_{n}, n=1,2, \ldots$, be an increasing sequence of positive integers (by "increasing" we mean strictly increasing). The double sequence $x_{m} / x_{n}, m, n=1,2, \ldots$. is called the ratio sequence of $x_{n}$. It was introduced by T. S a lát [16]. He studied its everywhere density. For further study of the ratio sequences, O. S t r a u c h and J. T. Tóth [24] introduced a sequence $X_{n}$ of blocks

$$
X_{n}=\left(\frac{x_{1}}{x_{n}}, \frac{x_{2}}{x_{n}}, \ldots, \frac{x_{n}}{x_{n}}\right), \quad n=1,2, \ldots
$$

(C) 2015 Mathematical Institute, Slovak Academy of Sciences.

2010 Mathematics Subject Classification: 11K31, $11 \mathrm{~K} 38$.

Keywords: block sequence, distribution function, asymptotic density.

Supported by APVV Project SK-CZ-0075-11 and VEGA Project 2/0146/14. 


\section{OTO STRAUCH}

and they studied the set $G\left(X_{n}\right)$ of its distribution functions. The motivation is that the existence of strictly increasing $g(x) \in G\left(X_{n}\right)$ implies everywhere density of $x_{m} / x_{n}$, the basic problem studied by $\check{\mathrm{S}}$ a lát [16]. Further motivation is that the block sequences are a tool for study of distribution functions of sequences, see [20, p. 12, 1.9]. Organization of the paper:

In Section 2 we follow the notations and basic properties of distribution functions used in [5], 12] and [21, p. 1-28, 1.8.23].

In Section 3 we list main properties of $g(x)$ and $G\left(X_{n}\right)$ without proofs.

In Section 4 we add proofs of some properties in Section 3 . Specially:

4.1 Basic properties;

4.2 Continuity of $g(x) \in G\left(X_{n}\right)$;

4.3 Singleton $G\left(X_{n}\right)=\{g(x)\}$;

4.4 U.d. of $X_{n}$;

4.5 One-step d.f.s $c_{\alpha}(x)$;

4.6. Connectivity of $G\left(X_{n}\right)$;

4.7 Boundaries of $g(x) \in G\left(X_{n}\right)$;

4.8 Lower and upper d.f.s in $G\left(X_{n}\right)$;

4.9 Construction $H \subset G\left(X_{n}\right)$;

$4.10 g(x) \in G\left(X_{n}\right)$ with constant intervals;

4.11 Transformation of $X_{n}$ by $1 / x \bmod 1$.

Many examples with $x_{n}$ and $G\left(X_{n}\right)$ are given in Section 5. The paper is completed in Section [ with comments on another block sequences.

\section{Definitions}

- From now on $1 \leq x_{1}<x_{2}<\cdots$ denotes the sequence of positive integers and $x \in[0,1)$.

- Denote by $F\left(X_{n}, x\right)$ the step distribution function

$$
F\left(X_{n}, x\right)=\frac{\#\left\{i \leq n ; \frac{x_{i}}{x_{n}}<x\right\}}{n},
$$

for $x \in[0,1)$ and for $x=1$ we define $F\left(X_{n}, 1\right)=1$.

- Denote by $A(t)$ the counting function

$$
A(t)=\#\left\{n \in \mathbb{N} ; x_{n}<t\right\} .
$$




\section{DISTRIBUTION FUNCTIONS OF RATIO SEQUENCES. AN EXPOSITORY PAPER}

Directly from the definition we obtain

$$
F\left(X_{m}, x\right)=\frac{n}{m} F\left(X_{n}, x \frac{x_{m}}{x_{n}}\right)
$$

for each $m \leq n$ and

$$
\frac{n F\left(X_{n}, x\right)}{x x_{n}}=\frac{A\left(x x_{n}\right)}{x x_{n}}
$$

for every $x \in[0,1)$.

- The lower asymptotic density $\underline{d}$ and the upper asymptotic density $\bar{d}$ of $x_{n}$, $n=1,2, \ldots 1$ are defined as

$$
\underline{d}=\liminf _{t \rightarrow \infty} \frac{A(t)}{t}=\liminf _{n \rightarrow \infty} \frac{n}{x_{n}}, \quad \bar{d}=\limsup _{t \rightarrow \infty} \frac{A(t)}{t}=\limsup _{n \rightarrow \infty} \frac{n}{x_{n}} .
$$

- A non-decreasing function $g:[0,1] \rightarrow[0,1], g(0)=0, g(1)=1$ is called distribution function (abbreviated d.f.). We shall identify any two d.f.s coinciding at common points of continuity.

- Similarly, the inequality $g_{1}(x) \leq g_{2}(x)$ we consider only in the common points of continuity.

- A d.f. $g(x)$ is a d.f. of the sequence of blocks $X_{n}, n=1,2, \ldots$, if there exists an increasing sequence $n_{1}<n_{2}<\cdots$ of positive integers such that

$$
\lim _{k \rightarrow \infty} F\left(X_{n_{k}}, x\right)=g(x)
$$

a.e. on $[0,1]$. This is equivalent to the weak convergence, i.e., the preceding limit holds for every point $x \in[0,1]$ of continuity of $g(x)$.

- Denote by $G\left(X_{n}\right)$ the set of all d.f.s of $X_{n}, n=1,2, \ldots$ If $G\left(X_{n}\right)=\{g(x)\}$ is a singleton, the d.f. $g(x)$ is also called the asymptotic d.f. (abbreviated a.d.f.) of $X_{n}$.

- Also for a sequence $y_{n} \in[0,1), n=1,2, \ldots$, we have defined in [21, 1.3] the step d.f.

$$
F_{N}(x)=\frac{\#\left\{n \leq N ; y_{n} \in[0, x)\right\}}{N}
$$

and $G\left(y_{n}\right)$ is the set of all possible weak limits $F_{N_{k}}(x) \rightarrow g(x)$.

- The lower d.f. $\underline{g}(x)$ and the upper d.f. $\bar{g}(x)$ of a sequence $X_{n}, n=1,2, \ldots$ are defined as

$$
\underline{g}(x)=\inf _{g \in G\left(X_{n}\right)} g(x), \quad \bar{g}(x)=\sup _{g \in G\left(X_{n}\right)} g(x) .
$$

$\overline{1_{\underline{d}}=\underline{d}\left(x_{n}\right), \bar{d}}=\bar{d}\left(x_{n}\right)$. 
- If $\lim _{k \rightarrow \infty} F\left(X_{n_{k}}, x\right)=g(x)$ and $\lim _{k \rightarrow \infty} \frac{n_{k}}{x_{n_{k}}}=d_{g}$ we shall call $d_{g}$ as a local asymptotic density for d.f. $g(x)$.

In this paper we frequently use the following two theorems of Helly (see the First and Second Helly theorem [21, Th. 4.1.0.10 and Th. 4.1.0.11, p. 4-5]).

- Helly's selection principle: For any sequence $g_{n}(x), n=1,2, \ldots$, of d.f.s in $[0,1]$ there exists a subsequence $g_{n_{k}}(x), k=1,2, \ldots$, and a d.f. $g(x)$ such that $\lim _{k \rightarrow \infty} g_{n_{k}}(x)=g(x)$ a.e.

- Second Helly theorem: If we have $\lim _{n \rightarrow \infty} g_{n}(x)=g(x)$ a.e. in $[0,1]$, then for every continuous function $f:[0,1] \rightarrow \mathbb{R}$ we have $\lim _{n \rightarrow \infty} \int_{0}^{1} f(x) \mathrm{d} g_{n}(x)=$ $\int_{0}^{1} f(x) \mathrm{d} g(x)$.

- Note that applying Helly's selection principle, from the sequence $F\left(X_{n}, x\right)$, $n=1,2, \ldots$, one can select a subsequence $F\left(X_{n_{k}}, x\right), k=1,2, \ldots$, such that $\lim _{k \rightarrow \infty} F\left(X_{n_{k}}, x\right)=g(x)$ holds not only for the continuity points $x$ of $g(x)$, but also for all $x \in[0,1]$.

- We will use the one-step d.f. $c_{\alpha}(x)$ with the step 1 at $\alpha$ defined on $[0,1]$ via

$$
c_{\alpha}(x)= \begin{cases}0, & \text { if } x \leq \alpha \\ 1, & \text { if } x>\alpha\end{cases}
$$

while always $c_{\alpha}(0)=0$ and $c_{\alpha}(1)=1$.

\section{Overview of basic results}

$G\left(X_{n}\right)$ has the following properties:

1. If $g(x) \in G\left(X_{n}\right)$ increases and is continuous at $x=\beta$ and $g(\beta)>0$, then there exists $1 \leq \alpha<\infty$ such that $\alpha g(x \beta) \in G\left(X_{n}\right)$. If every d.f. of $G\left(X_{n}\right)$ is continuous at 1 , then $\alpha=1 / g(\beta)$, 24, Prop. 3.1, Th. 3.2].

2. Assume that all d.f.s in $G\left(X_{n}\right)$ are continuous at 0 and $c_{1}(x) \notin G\left(X_{n}\right)$. Then for every $\tilde{g}(x) \in G\left(X_{n}\right)$ and every $1 \leq \alpha<\infty$ there exists $g(x) \in$ $G\left(X_{n}\right)$ and $0<\beta \leq 1$ such that $\tilde{g}(x)=\alpha g(x \beta)$ a.e. [24, Th. 3.3].

3. Assume that all d.f.s in $G\left(X_{n}\right)$ are continuous at 1. Then all d.f.s in $G\left(X_{n}\right)$ are continuous on $(0,1]$, i.e., only possible discontinuity is in 0 [24, Th. 4.1].

4. If $\underline{d}\left(x_{n}\right)>0$, then every $g(x) \in G\left(X_{n}\right)$ is continuous on $[0,1]$, [24, Th. 6.2(iv)].

5. If $\underline{d}\left(x_{n}\right)>0$, then there exists $g(x) \in G\left(X_{n}\right)$ such that $g(x) \geq x$ for every $x \in[0,1]$, 24, Th. 6.2(ii)]. Generally, [3, Th. 6)], every $G\left(X_{n}\right)$ contains $g(x) \geq x$ for every $x \in[0,1]$. 


\section{DISTRIBUTION FUNCTIONS OF RATIO SEQUENCES. AN EXPOSITORY PAPER}

6. If $\bar{d}\left(x_{n}\right)>0$, then there exists $g(x) \in G\left(X_{n}\right)$ such that $g(x) \leq x$ for every $x \in[0,1]$, 24, Th. 6.2].

7. Assume that $G\left(X_{n}\right)$ is singleton, i.e., $G\left(X_{n}\right)=\{g(x)\}$. Then either $g(x)=$ $c_{0}(x)$ for $x \in[0,1]$; or $g(x)=x^{\lambda}$ for some $0<\lambda \leq 1$ and $x \in[0,1]$. Moreover, if $\bar{d}\left(x_{n}\right)>0$, then $g(x)=x$, [24, Th. 8.2].

8. $\max _{g \in G\left(X_{n}\right)} \int_{0}^{1} g(x) \mathrm{d} x \geq \frac{1}{2}$, [24, Th. 7.1] (c.f. 5.).

9. Assume that every d.f. $g(x) \in G\left(X_{n}\right)$ has a constant value on the fixed interval $(u, v) \subset[0,1]$ (maybe different). If $\underline{d}\left(x_{n}\right)>0$ then all d.f.s in $G\left(X_{n}\right)$ has infinitely many intervals with constant values, 22 .

10. There exists an increasing sequence $x_{n}, n=1,2, \ldots$, of positive integers such that $G\left(X_{n}\right)=\left\{h_{\alpha}(x) ; \alpha \in[0,1]\right\}$, where $h_{\alpha}(x)=\alpha, x \in(0,1)$ is the constant d.f. 9, Ex. 1].

11. There exists an increasing sequence $x_{n}, n=1,2, \ldots$, of positive integers such that $c_{1}(x) \in G\left(X_{n}\right)$ but $c_{0}(x) \notin G\left(X_{n}\right)$, where $c_{0}(x)$ and $c_{1}(x)$ are one-jump d.f.s with the jump of height 1 at $x=0$ and $x=1$, respectively.

12. There exists an increasing sequence $x_{n}, n=1,2, \ldots$, of positive integers such that $G\left(X_{n}\right)$ is non-connected [9, Ex. 2].

13. We have (see [24, Prop. 3.1, Th. 3.2]):

Let $g(x) \in G\left(X_{n}\right), \beta \in(0,1)$, and assuming that

(i) $g(x)$ is continuous at $\beta$,

(ii) $g(x)$ increases at $\beta 2$

(iii) $g(\beta)>0$,

(iv) all d.f. in $G\left(X_{n}\right)$ are continuous at 1.

Then

$$
\frac{g(x \beta)}{g(\beta)} \in G\left(X_{n}\right) .
$$

14. Taking the following limits (i)-(iii) for a sequence of indices $n_{k}, k=1,2, \ldots$

(i) $\lim _{k \rightarrow \infty} F\left(X_{n_{k}}, x\right)=g(x)$,

(ii) $\lim _{k \rightarrow \infty} \frac{n_{k}}{x_{n_{k}}}=d_{g}$,

then (see [24, Prop. 6.1]) there exists

(iii) $\lim _{k \rightarrow \infty} \frac{A\left(x x_{n_{k}}\right)}{x x_{n_{k}}}=d_{g}(x)$ and

$$
\frac{g(x)}{x} d_{g}=d_{g}(x)
$$

for $x \in[0,1]$. Here the limits (i) and (iii) can be considered for all $x \in(0,1]$ or all continuity points $x \in(0,1]$ of $g(x)$ and the constant $d_{g}$ in (ii) we call local density.

\footnotetext{
${ }^{2}$ The assumption (ii) can be replaced by a requirement that $\beta$ is a limit point of $\frac{x_{i}}{x_{n_{k}}}$, $i=1,2, \ldots, n_{k}, k=1,2, \ldots$, where weakly $F\left(X_{n_{k}}, x\right) \rightarrow g(x)$.
} 
15. Specially (see [24, Th. 6.2 (iii), (iv)]), if $\underline{d}>0$ then

$$
x \frac{\underline{d}}{\bar{d}} \leq g(x) \leq x \frac{\bar{d}}{\underline{d}}
$$

for every $x \in[0,1]$ and furthermore $g(x)$ is everywhere continuous. Thus $\underline{d}=\bar{d}>0$ implies u.d. of the block sequence $X_{n}, n=1,2, \ldots$

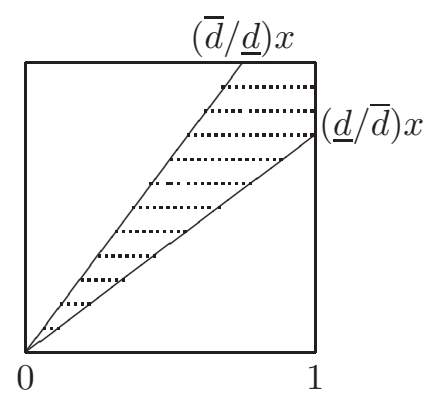

16. $G\left(X_{n}\right)=\left\{x^{\lambda}\right\}$ if and only if $\lim _{n \rightarrow \infty}\left(x_{k . n} / x_{n}\right)=k^{1 / \lambda}$ for every $k=1,2, \ldots$ Here as in 7 . we have $0<\lambda \leq 1,7$.

17. If $\underline{d}\left(x_{n}\right)>0$, then all d.f.s $g(x) \in G\left(X_{n}\right)$ are continuous, nonsingular and bounded by $h_{1}(x) \leq g(x) \leq h_{2}(x)$, where

$$
h_{1}(x)= \begin{cases}x \frac{d}{\bar{d}} & \text { if } x \in\left[0, \frac{1-\bar{d}}{1-\underline{d}}\right], \quad h_{2}(x)=\min \left(x \frac{\bar{d}}{\underline{d}}, 1\right) . \\ \frac{\underline{d}}{\frac{1}{x}-(1-\underline{d})} & \text { otherwise, }\end{cases}
$$

Furthermore, there exists $x_{n}, n=1,2, \ldots$, such that $h_{2}(x) \in G\left(X_{n}\right)$ and for every $x_{n}$ we have $h_{1}(x) \notin G\left(X_{n}\right)$, [3, Th. 7] and moreover

18. for a given fixed $g(x) \in G\left(X_{n}\right), x \in[0,1]$ we have $h_{1, g}(x) \leq g(x) \leq h_{2, g}(x)$, where

$$
\begin{aligned}
& h_{1, g}(x)= \begin{cases}x \frac{d}{d_{g}} & \text { if } x<y_{0}=\frac{1-d_{g}}{1-\underline{d}} \\
x \frac{1}{d_{g}}+1-\frac{1}{d_{g}} & \text { if } y_{0} \leq x \leq 1\end{cases} \\
& h_{2, g}(x)=\min \left(x \frac{\bar{d}}{d_{g}}, 1\right)
\end{aligned}
$$

[3. Th. 6].

19. These boundaries are established by observing that for every $g(x) \in G\left(X_{n}\right)$

$$
0 \leq \frac{g(y)-g(x)}{y-x} \leq \frac{1}{d_{g}}
$$

for $x<y, x, y \in[0,1]$. 


\section{DISTRIBUTION FUNCTIONS OF RATIO SEQUENCES. AN EXPOSITORY PAPER}

\section{Overview of proofs}

In this section we give proofs of some properties described in Section 3 .

\subsection{Basic properties}

Using

$$
x_{i}<x x_{m} \Longleftrightarrow x_{i}<\left(x \frac{x_{m}}{x_{n}}\right) x_{n}
$$

and that these inequalities imply $i<m$, it directly follows from definition $F\left(X_{n}, x\right)$ that

$$
F\left(X_{m}, x\right)=\frac{n}{m} F\left(X_{n}, x \frac{x_{m}}{x_{n}}\right),
$$

for every $m \leq n$ and $x \in[0,1)$. Also for any increasing sequence of positive integers $x_{n}, n=1,2, \ldots$, we define a counting function $A(t)$ as

$$
A(t)=\#\left\{n \in \mathbb{N} ; x_{n}<t\right\} .
$$

Then for every $x \in(0,1]$ we have the equality

$$
\frac{n F\left(X_{n}, x\right)}{x x_{n}}=\frac{A\left(x x_{n}\right)}{x x_{n}},
$$

which we shall use to compute the asymptotic density of $x_{n}$. We have the lower asymptotic density $\underline{d}$, and the upper asymptotic density $\bar{d}$ of $x_{n}, n=1,2, \ldots$ as

$$
\underline{d}=\liminf _{t \rightarrow \infty} \frac{A(t)}{t}=\liminf _{n \rightarrow \infty} \frac{n}{x_{n}}, \quad \bar{d}=\limsup _{t \rightarrow \infty} \frac{A(t)}{t}=\limsup _{n \rightarrow \infty} \frac{n}{x_{n}} .
$$

Using Helly's selection principle from the sequence $(m, n)$ we can select a subsequence $\left(m_{k}, n_{k}\right)$ such that $F\left(X_{n_{k}}\right) \rightarrow g(x), F\left(X_{m_{k}}\right) \rightarrow \tilde{g}(x)$ as $k \rightarrow \infty$, furthermore $x_{m_{k}} / x_{n_{k}} \rightarrow \beta$ and $m_{k} / n_{k} \rightarrow \alpha$, but $\alpha$ may be infinity. These limits have the following connection.

Theorem 1 ([24, Prop. 3.1]). Let $m_{k}$ and $n_{k}$ be two increasing integer sequences satisfying $m_{k} \leq n_{k}$, for $k=1,2, \ldots$ and assume that

(i) $\lim _{k \rightarrow \infty} F\left(X_{n_{k}}, x\right)=g(x)$ a.e.,

(ii) $\lim _{k \rightarrow \infty} F\left(X_{m_{k}}, x\right)=\tilde{g}(x)$ a.e.,

(iii) $\lim _{k \rightarrow \infty} \frac{x_{m_{k}}}{x_{n_{k}}}=\beta>0$,

(iv) $g(\beta-0)>0$.

Then there exists $\lim _{k \rightarrow \infty} \frac{n_{k}}{m_{k}}=\alpha<\infty$ such that

$$
\tilde{g}(x)=\alpha g(x \beta) \quad \text { a.e. on }[0,1], \quad \text { and } \quad \alpha=\frac{\tilde{g}(1-0)}{g(\beta-0)} \text {. }
$$




\section{OTO STRAUCH}

Pr o of. Firstly we prove

$$
\lim _{k \rightarrow \infty} F\left(X_{n_{k}}, x \frac{x_{m_{k}}}{x_{n_{k}}}\right)=g(x \beta) .
$$

Denoting $\beta_{k}=x_{m_{k}} / x_{n_{k}}$ and substituting $u=x \beta_{k}$, we find

$$
\begin{aligned}
0 \leq \int_{0}^{1}\left(F\left(X_{n_{k}}, x \beta_{k}\right)-g\left(x \beta_{k}\right)\right)^{2} \mathrm{~d} x & =\frac{1}{\beta_{k}} \int_{0}^{\beta_{k}}\left(F\left(X_{n_{k}}, u\right)-g(u)\right)^{2} \mathrm{~d} u \\
& \leq \frac{1}{\beta_{k}} \int_{0}^{1}\left(F\left(X_{n_{k}}, u\right)-g(u)\right)^{2} \mathrm{~d} u \rightarrow 0,
\end{aligned}
$$

which leads to $\left(F\left(X_{n_{k}}, x \beta_{k}\right)-g\left(x \beta_{k}\right)\right) \rightarrow 0$ a.e. as $k \rightarrow \infty$ (here necessarily $\beta>0)$. Furthermore,

$$
\begin{aligned}
& \int_{0}^{1}\left(F\left(X_{n_{k}}, x \beta_{k}\right)-g(x \beta)\right)^{2} \mathrm{~d} x \\
& =\int_{0}^{1}\left(F\left(X_{n_{k}}, x \beta_{k}\right)-g\left(x \beta_{k}\right)+g\left(x \beta_{k}\right)-g(x \beta)\right)^{2} \mathrm{~d} x \\
& \leq 2\left(\int_{0}^{1}\left(F\left(X_{n_{k}}, x \beta_{k}\right)-g\left(x \beta_{k}\right)\right)^{2} \mathrm{~d} x+\int_{0}^{1}\left(g\left(x \beta_{k}\right)-g(x \beta)\right)^{2} \mathrm{~d} x\right) .
\end{aligned}
$$

Since $g(x)$ is continuous a.e. on $[0,1]$ then $\left(g\left(x \beta_{k}\right)-g(x \beta)\right) \rightarrow 0$ a.e. and applying the Lebesgue theorem of dominant convergence we find $\int_{0}^{1}\left(g\left(x \beta_{k}\right)-\right.$ $g(x \beta))^{2} \mathrm{~d} x \rightarrow 0$. This gives (44). The existence of the $\operatorname{limit}_{k \rightarrow \infty} \frac{n_{k}}{m_{k}}=\alpha<\infty$ follows from (1) and (iv). Now, let $t_{n} \in[0,1)$ increases to 1 and $\tilde{g}(x)$ be continuous in $t_{n}$. Then $g(x \beta)$ is also continuous in $t_{n}$ and $\tilde{g}\left(t_{n}\right)=\alpha g\left(t_{n} \beta\right)$ for $n=1,2, \ldots$ The limit of this equation gives the desired form of $\alpha$.

The equality (2) gives

Theorem 2 ([24, Prop. 6.1]). Assume for a sequence $n_{k}, k=1,2, \ldots$ that

(i) $\lim _{k \rightarrow \infty} F\left(X_{n_{k}}, x\right)=g(x)$,

(ii) $\lim _{k \rightarrow \infty} \frac{n_{k}}{x_{n_{k}}}=d_{g}$.

Then there exists

(iii) $\lim _{k \rightarrow \infty} \frac{A\left(x x_{n_{k}}\right)}{x x_{n_{k}}}=d_{g}(x)$ and

$$
g(x)=\frac{x}{d_{g}} d_{g}(x)
$$

Here the limits (i) and (iii) can be considered for all $x \in(0,1]$ or all continuity points $x \in(0,1]$ of $g(x)$. 


\section{DISTRIBUTION FUNCTIONS OF RATIO SEQUENCES. AN EXPOSITORY PAPER}

\subsection{Continuity of $g \in G\left(X_{n}\right)$}

If all $g \in G\left(X_{n}\right)$ are everywhere continuous on [0,1], then relation (3) is of the form

$$
\frac{g(x \beta)}{g(\beta)} \in G\left(X_{n}\right) .
$$

As a criterion for continuity of all $g \in G\left(X_{n}\right)$ we can adapt the Wiener-Schoenberg theorem (cf. [12, 6, p. 55]), but here we give the following simple sufficient condition.

Theorem 3 ([24, Th. 4.1]). Assume that all d.f.s in $G\left(X_{n}\right)$ are continuous at 1. Then all d.f.s in $G\left(X_{n}\right)$ are continuous on $(0,1]$, i.e., the only discontinuity point can be 0 .

P r o of. Assume that $x_{m_{k}} / x_{n_{k}} \rightarrow \beta$ and $F\left(X_{n_{k}}, x\right) \rightarrow g(x)$ as $k \rightarrow \infty$. If from $\left(m_{k}, n_{k}\right)$ we can select two sequences $\left(m_{k}^{\prime}, n_{k}^{\prime}\right)$ and $\left(m_{k}^{\prime \prime}, n_{k}^{\prime \prime}\right)$ such that $n_{k}^{\prime} / m_{k}^{\prime} \rightarrow \alpha_{1}$ and $n_{k}^{\prime \prime} / m_{k}^{\prime \prime} \rightarrow \alpha_{2}$ with a finite $\alpha_{1} \neq \alpha_{2}$, then $\alpha_{1} g(x \beta), \alpha_{2} g(x \beta) \in$ $G\left(X_{n}\right)$ and thus one of such d.f. $\tilde{g}(x)$ must be discontinuous at 1 (it holds also for $g$ continuous at $\beta$ ). Thus, assuming that $G\left(X_{n}\right)$ has only continuous d.f.s at 1 , the limits $x_{m_{k}} / x_{n_{k}} \rightarrow \beta>0$ and $F\left(X_{n_{k}}, x\right) \rightarrow g(x)$ imply the convergence of $n_{k} / m_{k}$. Now by [24, Th. 3.2]: If $\beta$ is a point of discontinuity of $g(x)$ with $g(\beta+0)-g(\beta-0)=h>0$, then there exists a closed interval $I \subset[0,1]$, with length $|I| \geq h$ such that for every $\frac{1}{\alpha} \in I$ we have $\alpha g(x \beta) \in G\left(X_{n}\right)$. Thus $g(x)$ cannot have a discontinuity point in $(0,1]$.

Theorem 4 ([24, Th. 6.2]).

(i) If $\bar{d}>0$, then there exits $g \in G\left(X_{n}\right)$ such that $g(x) \leq x$ for every $x \in[0,1]$.

(ii) If $\underline{d}>0$, then there exits $g \in G\left(X_{n}\right)$ such that $g(x) \geq x$ for every $x \in[0,1]$.

(iii) If $\underline{d}>0$, then for every $g \in G\left(X_{n}\right)$ we have

$$
(\underline{d} / \bar{d}) x \leq g(x) \leq(\bar{d} / \underline{d}) x
$$

for every $x \in[0,1]$.

(iv) If $\underline{d}>0$, then every $g \in G\left(X_{n}\right)$ is everywhere continuous in $[0,1]$.

(v) If $\underline{d}>0$, then for every limit point $\beta>0$ of $x_{m} / x_{n}$ there exist $g \in G\left(X_{n}\right)$ and $0 \leq \alpha<\infty$ such that $\alpha g(x \beta) \in G\left(X_{n}\right)$.

Proof. (i). Assume that $n_{k} / x_{n_{k}} \rightarrow \bar{d}$ as $k \rightarrow \infty$. Select a subsequence $n_{k}^{\prime}$ of $n_{k}$ such that $F\left(X_{n_{k}^{\prime}}, x\right) \rightarrow g(x)$ a.e. on $[0,1]$. Since $d_{g}(x) \leq \bar{d}$ a.e. in (5) gives $(g(x) / x) \bar{d} \leq \bar{d}$ a.e., which leads to $g(x) \leq x$ a.e. and implies $g(x) \leq x$ for every $x \in[0,1]$. 


\section{OTO STRAUCH}

(ii). Similarly to (i), let $n_{k} / x_{n_{k}} \rightarrow \underline{d}$ as $k \rightarrow \infty$. Select a subsequence $n_{k}^{\prime}$ of $n_{k}$ such that $F\left(X_{n_{k}^{\prime}}, x\right) \rightarrow g(x)$ a.e. on $[0,1]$. Since $d_{2}(x) \geq \underline{d}$ a.e., (5]) implies $(g(x) / x) \underline{d} \geq \underline{d}$ a.e. again, which gives $g(x) \geq x$ a.e., whence, $g(x) \geq x$ everywhere on $x \in[0,1]$.

(iii). For any $g \in G\left(X_{n}\right)$ there exists $n_{k}$ such that $F\left(X_{n_{k}}, x\right) \rightarrow g(x)$ a.e. From $n_{k}$ we can choose a subsequence $n_{k}^{\prime}$ such that $n_{k}^{\prime} / x_{n_{k}^{\prime}} \rightarrow d_{1}$. Using (5) and the fact that $\underline{d} \leq d_{1} \leq \bar{d}$ and $\underline{d} \leq d_{2} \leq \bar{d}$ we have $(g(x) / x) \underline{d} \leq \bar{d}$ and $(g(x) / x) \bar{d} \geq \underline{d}$ a.e. If $\underline{d}>0$, these inequalities are valid for every $x \in(0,1]$.

(iv). Continuity of $g \in G\left(X_{n}\right)$ at 1 follows from [24, Prop. 4.2]: Denote

$$
\bar{d}(\varepsilon)=\limsup _{n \rightarrow \infty} \frac{\#\left\{i \leq n ;(1-\varepsilon) x_{n}<x_{i}<x_{n}\right\}}{n} .
$$

Every $g \in G\left(X_{n}\right)$ is continuous at 1 if and only if $\lim _{\varepsilon \rightarrow 0} \bar{d}(\varepsilon)=0$. Since

$$
\bar{d}(\varepsilon) \leq \limsup _{n \rightarrow \infty} \varepsilon \frac{x_{n}}{n}=\frac{\varepsilon}{\underline{d}},
$$

applying [24, Th. 4.1] = Theorem [3, we have continuity of $g$ in $(0,1]$. Continuity at 0 follows from (7).

(v). It follows from the fact that if $\underline{d}>0$ and $\lim _{k \rightarrow \infty} x_{m_{k}} / x_{n_{k}}=\beta>0$ for $m_{k}<n_{k}$, then $\lim \sup _{k \rightarrow \infty} n_{k} / m_{k}<\infty$. More precisely, if we pick $\left(m_{k}^{\prime}, n_{k}^{\prime}\right)$ from $\left(m_{k}, n_{k}\right)$ such that $n_{k}^{\prime} / m_{k}^{\prime} \rightarrow \alpha$, then

$$
\frac{\underline{d}}{\bar{d} \beta} \leq \alpha \leq \frac{\bar{d}}{\underline{d} \beta} .
$$

This is so because if we select $\left(m_{k}^{\prime \prime}, n_{k}^{\prime \prime}\right)$ from $\left(m_{k}^{\prime}, n_{k}^{\prime}\right)$ such that $n_{k}^{\prime \prime} / x_{n_{k}^{\prime \prime}} \rightarrow d_{1}$ and $m_{k}^{\prime \prime} / x_{m_{k}^{\prime \prime}} \rightarrow d_{2}$, then, by

we see $\alpha=d_{1} /\left(d_{2} \beta\right)$.

$$
\frac{n_{k}^{\prime \prime}}{m_{k}^{\prime \prime}}=\frac{\frac{n_{k}^{\prime \prime}}{x_{n_{k}^{\prime \prime}}} x_{n_{k}^{\prime \prime}}}{\frac{m_{k}^{\prime \prime}}{x_{m_{k}^{\prime \prime}}} x_{m_{k}^{\prime \prime}}},
$$

\subsection{Singleton $G\left(X_{n}\right)=\{g\}$}

For general $G\left(X_{n}\right)$, the connection between $G\left(X_{n}\right)$ and $G\left(x_{m} / x_{n} \bmod 1\right)$ is open, but for singleton $G\left(X_{n}\right)$ we have

Theorem 5 ([24, Th. 8.1]). If $G\left(X_{n}\right)=\{g\}$, then $G\left(x_{m} / x_{n} \bmod 1\right)=\{g\}$.

Proof. A proof of the theorem is the same as the proof of [19, Prop. 1, (ii)], since

$$
\lim _{n \rightarrow \infty} \frac{\left|X_{n}\right|}{\left|X_{1}\right|+\cdots+\left|X_{n}\right|}=\lim _{n \rightarrow \infty} \frac{n}{n(n+1) / 2}=0 .
$$


Theorem 6 ([24, Th. 8.2]). Assume that $G\left(X_{n}\right)=\{g\}$. Then either

(i) $g(x)=c_{0}(x)$ for $x \in[0,1]$ or

(ii) $g(x)=x^{\lambda}$ for some $0<\lambda \leq 1$ and $x \in[0,1]$. Moreover,

(iii) if $\bar{d}>0$ then $g(x)=x$.

P r o of. Let $G\left(X_{n}\right)=\{g\}$. We divide the proof into the following six steps.

(I). By [24, Th. 7.1], we have $\int_{0}^{1} g(x) \mathrm{d} x \geq \frac{1}{2}$ which implies $g(x) \neq c_{1}(x)$.

(II). $g$ must be continuous on $(0,1)$, since otherwise [24, Th. 3.2], for a discontinuity point $\beta \in(0,1)$, guarantees the existence of $\alpha_{1} \neq \alpha_{2}$ such that $\alpha_{1} g(x \beta)=\alpha_{2} g(x \beta)=g(x)$ a.e. which is a contradiction.

(III). Assume that $g(x)$ increases in every point $\beta \in(0,1)$. In this case relation (5) gives the well-known Cauchy equation $g(x) g(\beta)=g(x \beta)$ for a.e. $x, \beta \in[0,1]$ For a monotonic $g(x)$ the Cauchy equation has solutions only of the type $g(x)=x^{\lambda}$.

(IV). Assume that $g(x)$ has a constant value on the interval $(\gamma, \delta) \subset[0,1]$. For $\beta \in(0,1] g(x)$ satisfies two conditions: (j) $g(x)$ increases in $\beta$ and $(\mathrm{jj})$ $g(\beta)>0$. Then the basic relation (3) gives $g(x)=\alpha g(x \beta)$ which implies that $g(x)$ has a constant value also on $\beta(\gamma, \delta)$ and if $\delta \leq \beta$ then also on $\beta^{-1}(\gamma, \delta)$. Thus, if $\left(\gamma_{i}, \delta_{i}\right), i \in \mathcal{I}$ is a system of all intervals (maximal under inclusion) in which $g(x)$ possesses constant values, then for every $i \in \mathcal{I}$ there exists $j \in \mathcal{I}$ such that $\beta\left(\gamma_{i}, \delta_{i}\right)=\left(\gamma_{j}, \delta_{j}\right)$ and vice-versa for every $j \in \mathcal{I}, \delta_{j} \leq \beta$, there exists $i \in \mathcal{I}$ such that $\beta^{-1}\left(\gamma_{j}, \delta_{j}\right)=\left(\gamma_{i}, \delta_{i}\right)$. This is true also for $\beta=\beta_{1}^{n_{1}} \beta_{2}^{n_{2}} \ldots$, where $\beta_{1}, \beta_{2}, \ldots$ satisfy $(\mathrm{j})$ and $(\mathrm{jj})$ and $n_{1}, n_{2}, \ldots \in \mathbb{Z}$. Thus, there exists $0<\theta<1$ such that every such $\beta$ has the form $\theta^{n}, n \in \mathbb{N}$. The end points $\gamma_{i}, \delta_{i}$ (without $\gamma_{i}=0$ ) satisfy $(\mathrm{j})$ and $(\mathrm{jj})$ and thus the intervals $\left(\gamma_{i}, \delta_{i}\right)$ is of the form $\left(\theta^{n}, \theta^{n-1}\right), n=1,2, \ldots$ and all discontinuity points of $g(x)$ are $\theta^{n}, n=1,2, \ldots$, a contradiction with (II). For $g(x)=c_{0}(x)$ there exists no $\beta \in(0,1]$ satisfying (j) and (jj).

$(\mathrm{V})$. We have the possibilities $g(x)=c_{0}(x)$ and $g(x)=x^{\lambda}$ for some $\lambda>0$. Applying [24, Th. 7.1] we have $\int_{0}^{1} g(x) \mathrm{d} x \geq 1 / 2$ which reduces $\lambda$ to $\lambda \leq 1$.

(VI). If $\bar{d}>0$, then by [24, Th. 6.2, (i)] = Theorem 4 must be $g(x) \leq x$ which is contrary to $x^{\lambda}>x$ for $\lambda<1$.

The possibilities (i), (ii) are achievable. Trivially, for $x_{n}=\left[n^{\lambda}\right], G\left(X_{n}\right)=$ $\left\{x^{1 / \lambda}\right\}$ and for $x_{n}$ satisfying $\lim _{n \rightarrow \infty} x_{n} / x_{n+1}=0$ we have $G\left(X_{n}\right)=\left\{c_{0}(x)\right\}$. Less trivially, every lacunary $x_{n}$, i.e., $x_{n} / x_{n+1} \leq \lambda<1$, gives $G\left(X_{n}\right)=\left\{c_{0}(x)\right\}$.

The following limit covers all of $G\left(X_{n}\right)=\{g\}$. 


\section{OTO STRAUCH}

Theorem 7 ([24, Th. 8.3]). The set $G\left(X_{n}\right)$ is a singleton if and only if

$$
\begin{aligned}
\lim _{m, n \rightarrow \infty}( & \frac{1}{m n} \sum_{i=1}^{m} \sum_{j=1}^{n}\left|\frac{x_{i}}{x_{m}}-\frac{x_{j}}{x_{n}}\right| \\
& \left.-\frac{1}{2 m^{2}} \sum_{i, j=1}^{m}\left|\frac{x_{i}}{x_{m}}-\frac{x_{j}}{x_{m}}\right|-\frac{1}{2 n^{2}} \sum_{i, j=1}^{n}\left|\frac{x_{i}}{x_{n}}-\frac{x_{j}}{x_{n}}\right|\right)=0 .
\end{aligned}
$$

P r o of. It follows directly from the limit (9) in the form

$$
\lim _{m, n \rightarrow \infty} \int_{0}^{1}\left(F\left(X_{m}, x\right)-F\left(X_{n}, x\right)\right)^{2} \mathrm{~d} x=0,
$$

after applying

$$
\begin{aligned}
\int_{0}^{1}(g(x)-\tilde{g}(x))^{2} \mathrm{~d} x= & \int_{0}^{1} \int_{0}^{1}|x-y| \mathrm{d} g(x) \mathrm{d} \tilde{g}(y) \\
& -\frac{1}{2} \int_{0}^{1} \int_{0}^{1}|x-y| \mathrm{d} g(x) \mathrm{d} g(y)-\frac{1}{2} \int_{0}^{1} \int_{0}^{1}|x-y| \mathrm{d} \tilde{g}(x) \mathrm{d} \tilde{g}(y)
\end{aligned}
$$

for $g(x)=F\left(X_{m}, x\right)$ and $\tilde{g}(x)=F\left(X_{n}, x\right)$.

\subsection{U.d. of $X_{n}$}

By Theorem [5, u.d. of the single block sequence $X_{n}$ implies the u.d. of the ratio sequence $x_{m} / x_{n}$. Applying [24, Th. 6.3, (i)] $(\underline{d} / \bar{d}) x \leq g(x) \leq(\bar{d} / \underline{d}) x$ for every $x \in[0,1]$, we have

TheOREM 8. If the increasing sequence $x_{n}$ of positive integers has a positive asymptotic density, i.e., $\underline{d}=\bar{d}>0$, then the associated ratio sequence $x_{m} / x_{n}$, $m=1,2, \ldots, n, n=1,2, \ldots$ is u.d. in $[0,1]$.

Positive asymptotic density is not necessary. According to T. Šalát [16] we can use also a sequence $x_{n}$ with $\underline{d}=0$.

TheORem 9 ([24, Th. 9.2]). Let $x_{n}$ be an increasing sequence of positive integers and $h:[0, \infty) \rightarrow[0, \infty)$ be a function satisfying

(i) $A(x) \sim h(x)$ as $x \rightarrow \infty$, where

(ii) $h(x y) \sim x h(y)$ as $y \rightarrow \infty$ and for every $x \in[0,1]$, and

(iii) $\lim _{n \rightarrow \infty} \frac{n}{h\left(x_{n}\right)}=1$.

Then $X_{n}$ (and consequently $x_{m} / x_{n}$ ) is u.d. in $[0,1]$. 
Proof. Starting with (2) $F\left(X_{n}, x\right) n=A\left(x x_{n}\right)$ it follows from (i) that

$$
\frac{F\left(X_{n}, x\right) n}{h\left(x x_{n}\right)} \rightarrow 1
$$

as $n \rightarrow \infty$, then by (ii)

$$
\frac{F\left(X_{n}, x\right) n}{x h\left(x_{n}\right)} \rightarrow 1
$$

which gives by (iii) the limit

as $n \rightarrow \infty$.

$$
F\left(X_{n}, x\right) \frac{n}{h\left(x_{n}\right)} \rightarrow x
$$

Assuming only (i) and (ii), we have $\liminf _{n \rightarrow \infty} n / h\left(x_{n}\right) \geq 1$, since otherwise $n_{k} / h\left(x_{n_{k}}\right) \rightarrow \alpha<1$ implies $F\left(X_{n_{k}}, x\right) \rightarrow x / \alpha$ for every $x \in[0,1]$ which is a contradiction. Also, $G\left(X_{n}\right) \subset\{x \lambda ; \lambda \in[0,1]\}$.

Another criterion can be found by using the so called $L^{2}$ discrepancy of the block $X_{n}$ defined by

$$
D^{(2)}\left(X_{n}\right)=\int_{0}^{1}\left(F\left(X_{n}, x\right)-x\right)^{2} \mathrm{~d} x,
$$

which can be expressed (cf. [19, IV. Appl.]) as

$$
D^{(2)}\left(X_{n}\right)=\frac{1}{n^{2}} \sum_{i, j=1}^{n} F\left(\frac{x_{i}}{x_{n}}, \frac{x_{j}}{x_{n}}\right),
$$

where

Thus

$$
F(x, y)=\frac{1}{3}+\frac{x^{2}+y^{2}}{2}-\frac{x+y}{2}-\frac{|x-y|}{2}
$$

$$
D^{(2)}\left(X_{n}\right)=\frac{1}{3}+\frac{1}{n x_{n}^{2}} \sum_{i=1}^{n} x_{i}^{2}-\frac{1}{n x_{n}} \sum_{i=1}^{n} x_{i}-\frac{1}{2 n^{2} x_{n}} \sum_{i, j=1}^{n}\left|x_{i}-x_{j}\right|,
$$

which gives (cf. [19]).

TheOREM 10. For every increasing sequence $x_{n}$ of positive integers we have

$$
\lim _{n \rightarrow \infty} D^{(2)}\left(X_{n}\right)=0 \Longleftrightarrow \lim _{n \rightarrow \infty} F\left(X_{n}, x\right)=x .
$$

The left hand-side can be divided into three limits (cf. [18, Th. 1])

$$
\lim _{n \rightarrow \infty} D^{(2)}\left(X_{n}\right)=0 \Longleftrightarrow\left\{\begin{array}{c}
(i) \lim _{n \rightarrow \infty} \frac{1}{n x_{n}} \sum_{i=1}^{n} x_{i}=\frac{1}{2}, \\
(\text { ii }) \lim _{n \rightarrow \infty} \frac{1}{n x_{n}^{2}} \sum_{i=1}^{n} x_{i}^{2}=\frac{1}{3}, \\
\left(\text { iii } \lim _{n \rightarrow \infty} \frac{1}{n^{2} x_{n}} \sum_{i, j=1}^{n}\left|x_{i}-x_{j}\right|=\frac{1}{3} .\right.
\end{array}\right.
$$

Weyl's criterion for u.d. of $X_{n}$ is not well applicable in our case. It says (cf. [17, (7)]). 
THEOREM 11. $X_{n}$ is u.d. if and only if

$$
\lim _{n \rightarrow \infty} \frac{1}{n} \sum_{k=1}^{n} e^{2 \pi i h \frac{x_{k}}{x_{n}}}=0
$$

for all positive integers $h$.

\subsection{One-step d.f. $c_{\alpha}(x)$}

In [24] there is proved that singleton $G\left(X_{n}\right)=\left\{c_{1}(x)\right\}$ does not exist, since (by [24, Th. 7.1]) for every increasing sequence $x_{n}$ of positive integers we have

$$
\max _{g(x) \in G\left(X_{n}\right)} \int_{0}^{1} g(x) \mathrm{d} x \geq \frac{1}{2} .
$$

In [24] is also proved (see Th. 8.4, 8.5) that

\section{Theorem 12.}

$$
\begin{gathered}
G\left(X_{n}\right)=\left\{c_{0}(x)\right\} \Longleftrightarrow \lim _{n \rightarrow \infty} \frac{1}{n x_{n}} \sum_{i=1}^{n} x_{i}=0 \\
G\left(X_{n}\right)=\left\{c_{0}(x)\right\} \Longleftrightarrow \lim _{n \rightarrow \infty} \frac{1}{m n} \sum_{i=1}^{m} \sum_{j=1}^{n}\left|\frac{x_{i}}{x_{m}}-\frac{x_{j}}{x_{n}}\right|=0, \\
G\left(X_{n}\right) \subset\left\{c_{\alpha}(x) ; \alpha \in[0,1]\right\} \Longleftrightarrow \lim _{n \rightarrow \infty} \frac{1}{n^{2} x_{n}} \sum_{i, j=1}^{n}\left|x_{i}-x_{j}\right|=0 .
\end{gathered}
$$

Proof.

(12). $\int_{0}^{1} x \mathrm{~d} g(x)=1-\int_{0}^{1} g(x) \mathrm{d} x=0$ only if $g(x)=c_{0}(x)$.

(13). Assume that $F\left(X_{m_{k}}, x\right) \rightarrow \tilde{g}(x)$ and $F\left(X_{n_{k}}, x\right) \rightarrow g(x)$ a.e. as $k \rightarrow \infty$.

Riemann-Stieltjes integration yields

$$
\frac{1}{m_{k} n_{k}} \sum_{i=1}^{m_{k}} \sum_{j=1}^{n_{k}}\left|\frac{x_{i}}{x_{m_{k}}}-\frac{x_{j}}{x_{n_{k}}}\right|=\int_{0}^{1} \int_{0}^{1}|x-y| \mathrm{d} F\left(X_{m_{k}}, x\right) \mathrm{d} F\left(X_{n_{k}}, y\right)
$$

which, after using Helly's theorem, tends to

$$
\int_{0}^{1} \int_{0}^{1}|x-y| \mathrm{d} \tilde{g}(x) \mathrm{d} g(y)
$$

as $k \rightarrow \infty$. Then (16) is equal to 0 if and only if $\tilde{g}(x)=g(x)=c_{\alpha}(x)$ for some fixed $\alpha \in[0,1]$. By Theorem 6 , $\alpha$ must be $0(\bar{d}=0$ follows from Theorem 4, part (i)). 


\section{DISTRIBUTION FUNCTIONS OF RATIO SEQUENCES. AN EXPOSITORY PAPER}

(14). Again $\int_{0}^{1} \int_{0}^{1}|x-y| \mathrm{d} g(x) \mathrm{d} g(y)=0$ if and only if $g(x)=c_{\alpha}(x)$ for $\alpha \in[0,1]$ and thus

$$
\lim _{k \rightarrow \infty} \frac{1}{n_{k} n_{k}} \sum_{i=1}^{n_{k}} \sum_{j=1}^{n_{k}}\left|\frac{x_{i}}{x_{n_{k}}}-\frac{x_{j}}{x_{n_{k}}}\right|=0
$$

for every $n_{k} \rightarrow \infty$. that

Furthermore, if $G\left(X_{n}\right) \subset\left\{c_{\alpha}(x) ; \alpha \in[0,1]\right\}$, then $\underline{d}\left(x_{n}\right)=0$. Here we prove

Theorem 13 ([9, Th. 6]). Let $x_{n}, n=1,2, \ldots$, be an increasing sequence of positive integers. Assume that $G\left(X_{n}\right) \subset\left\{c_{\alpha}(x) ; \alpha \in[0,1]\right\}$. Then $c_{0}(x) \in G\left(X_{n}\right)$ and if $G\left(X_{n}\right)$ contains two different d.f.s, then also $c_{1}(x) \in G\left(X_{n}\right)$.

Pr o of. We start from the equation (2) (see [24, p. 756, (1)])

$$
F\left(X_{m}, x\right)=\frac{n}{m} F\left(X_{n}, x \frac{x_{m}}{x_{n}}\right),
$$

which is valid for every $m \leq n$ and $x \in[0,1]$. Assuming, for two increasing sequences of indices $m_{k} \leq n_{k}$, that, as $k \rightarrow \infty$

(i) $F\left(X_{m_{k}}, x\right) \rightarrow c_{\alpha_{1}}(x)$ a.e.,

(ii) $F\left(X_{n_{k}}, x\right) \rightarrow c_{\alpha_{2}}(x)$ a.e.,

(iii) $\frac{n_{k}}{m_{k}} \rightarrow \gamma$,

(iv) $\frac{x_{m_{k}}}{x_{n_{k}}} \rightarrow \beta$,

(such sequences $m_{k} \leq n_{k}$ exist by Helly theorem) then we have:

a) If $\beta>0$ and $\gamma<\infty$ (see (3) in [24]), then

$$
c_{\alpha_{1}}(x)=\gamma c_{\alpha_{2}}(x \beta)
$$

for almost all $x \in[0,1]$.

b) If $\beta=0$ and $\gamma<\infty$, then by Helly theorem there exists subsequence $\left(m_{k}^{\prime}, n_{k}^{\prime}\right)$ of $\left(m_{k}, n_{k}\right)$ such that $F\left(X_{n_{k}^{\prime}}, x \frac{x_{m_{k}^{\prime}}}{x_{n_{k}^{\prime}}}\right) \rightarrow h(x)$ a.e. and since

$$
F\left(X_{n_{k}}, x \frac{x_{m_{k}^{\prime}}}{x_{n_{k}^{\prime}}}\right) \leq F\left(X_{n_{k}}, x \beta^{\prime}\right)
$$

for every $\beta^{\prime}>0$ and sufficiently large $k$, we get $h(x) \leq c_{\alpha_{2}}\left(x \beta^{\prime}\right)$. Summarizing, we have

$$
c_{\alpha_{1}}(x) \leq \gamma c_{\alpha_{2}}\left(x \beta^{\prime}\right)
$$

for every $\beta^{\prime}>0$ a.e. on $[0,1]$.

We distinguish the following steps (notions (i)-(iv), a) and b) are preserve): $1^{0}$. Let $c_{\alpha_{1}}(x) \in G\left(X_{n}\right), 0 \leq \alpha_{1}<1$, and let $m_{k}, k=1,2, \ldots$, be an increasing sequence of positive integers for which 


\section{OTO STRAUCH}

(i) $F\left(X_{m_{k}}, x\right) \rightarrow c_{\alpha_{1}}(x)$.

Relatively to the $m_{k}$, we choose an arbitrary sequence $n_{k}, m_{k} \leq n_{k}$, such that

(iii) $\frac{n_{k}}{m_{k}} \rightarrow \gamma, 1<\gamma<\infty$.

From $\left(m_{k}, n_{k}\right)$ we select a subsequence $\left(m_{k}^{\prime}, n_{k}^{\prime}\right)$ such that

(ii) $F\left(X_{n_{k}^{\prime}}, x\right) \rightarrow c_{\alpha_{2}}(x)$ a.e. on $[0,1]$,

(iv) $\frac{x_{m_{k}^{\prime}}}{x_{n_{k}^{\prime}}} \rightarrow \beta$ for some $\beta \in[0,1]$.

a) If $\beta>0$, then (13) $c_{\alpha_{1}}(x)=\gamma c_{\alpha_{2}}(x \beta)$ a.e. is impossible, because $\gamma>1$ and for $x>\alpha_{1}$ we have $c_{\alpha_{1}}(x)=1$. Thus $\beta=0$.

b) The condition $\beta=0$ implies (14) $c_{\alpha_{1}}(x) \leq \gamma c_{\alpha_{2}}\left(x \beta^{\prime}\right)$ for every $\beta^{\prime}>0$ and a.e. on $x \in[0,1]$. If $\alpha_{2}>0$, then $c_{\alpha_{2}}\left(x \beta^{\prime}\right)=0$ for all $x<\frac{\alpha_{2}}{\beta^{\prime}}$, which implies, using $\beta^{\prime} \leq \alpha_{2}$, that $c_{\alpha_{1}}(x)=0$ for $x \in(0,1)$, and this is contrary to the assumption $\alpha_{1}<1$.

Thus $\alpha_{2}=0$ and we have: If $0 \leq \alpha_{1}<1$ and $c_{\alpha_{1}}(x) \in G\left(X_{n}\right)$ then $c_{0}(x) \in G\left(X_{n}\right)$.

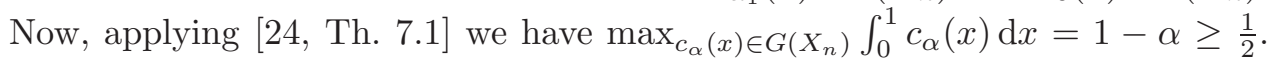
Then the assumption $c_{\alpha_{1}}(x) \in G\left(X_{n}\right), 0 \leq \alpha_{1}<1$ is true, thus $c_{0}(x) \in G\left(X_{n}\right)$ holds.

$2^{0}$ In this case we start with the sequence $n_{k}$ and we assume that $c_{\alpha_{2}}(x) \in G\left(X_{n}\right)$, $0<\alpha_{2} \leq 1$, and

(ii) $F\left(X_{n_{k}}, x\right) \rightarrow c_{\alpha_{2}}(x)$ a.e. on $[0,1]$.

Then we choose arbitrary $m_{k}$ such that $m_{k} \leq n_{k}$ and

(iii) $\frac{n_{k}}{m_{k}} \rightarrow \gamma, 1<\gamma<\infty$.

From $\left(m_{k}, n_{k}\right)$ we select a subsequence $\left(m_{k}^{\prime}, n_{k}^{\prime}\right)$ such that

(ii) $F\left(X_{m_{k}^{\prime}}, x\right) \rightarrow c_{\alpha_{1}}(x)$ a.e. on $[0,1]$,

(iv) $\frac{x_{m_{k}^{\prime}}}{x_{n_{k}^{\prime}}} \rightarrow \beta$ for some $\beta \in[0,1]$.

a) If $\beta>0$, then by (13) $c_{\alpha_{1}}(x)=\gamma c_{\alpha_{2}}(x \beta)$ a.e. If $\alpha_{1}<1$, then $\gamma>1$ implies $c_{\alpha_{1}}(x)>1$ for some $x \in(0,1)$, a contradiction. Thus $\alpha_{1}=1$ (in this case $\left.\beta \leq \alpha_{2}\right)$.

b) Now, $\beta=0$ implies (14) $c_{\alpha_{1}}(x) \leq \gamma c_{\alpha_{2}}\left(x \beta^{\prime}\right)$ for every $\beta^{\prime}>0$ and a.e. on $x \in[0,1]$ and the assumption $\alpha_{2}>0$ implies $c_{\alpha_{2}}\left(x \beta^{\prime}\right)=0$ for all $x<\frac{\alpha_{2}}{\beta^{\prime}}$, which gives $\alpha_{1}=1$. Summarizing, if $G\left(X_{n}\right)$ contains two different d.f.s, then it contains $c_{0}(x)$ and $c_{1}(x)$ simultaneously.

\subsection{Connectivity of $G\left(X_{n}\right)$}

As we have mentioned in the introduction, for a usual sequence $y_{n}$ the set $G\left(y_{n}\right)$ of all d.f. of $y_{n}$ is nonempty, closed and connected in the weak topology, 


\section{DISTRIBUTION FUNCTIONS OF RATIO SEQUENCES. AN EXPOSITORY PAPER}

and consists either of one or infinitely many functions. The closedness of $G\left(X_{n}\right)$ is clear, but connectivity of $G\left(X_{n}\right)$ is open. A general block sequence $Y_{n}$ with non-connected $G\left(Y_{n}\right)$ can be found trivially. For our special $X_{n}$ we have only the following sufficient condition.

Theorem 14 ([24, Th. 5.1]). If

$$
\begin{aligned}
\lim _{n \rightarrow \infty}\left(\frac{1}{n(n+1)} \sum_{i=1}^{n+1} \sum_{j=1}^{n}\left|\frac{x_{i}}{x_{n+1}}-\frac{x_{j}}{x_{n}}\right|\right. \\
\left.-\frac{1}{2(n+1)^{2}} \sum_{i, j=1}^{n+1}\left|\frac{x_{i}}{x_{n+1}}-\frac{x_{j}}{x_{n+1}}\right|-\frac{1}{2 n^{2}} \sum_{i, j=1}^{n}\left|\frac{x_{i}}{x_{n}}-\frac{x_{j}}{x_{n}}\right|\right)=0,
\end{aligned}
$$

then $G\left(X_{n}\right)$ is connected in the weak topology.

Proof. The connection follows from the limit

$$
\lim _{n \rightarrow \infty} \int_{0}^{1}\left(F\left(X_{n+1}, x\right)-F\left(X_{n}, x\right)\right)^{2} \mathrm{~d} x=0,
$$

since by a theorem of H. G. B a r on e 2] if $t_{n}$ is a sequence in a metric space $(X, \rho)$ satisfying

(i) any subsequence of $t_{n}$ contains a convergent subsequence and

(ii) $\lim _{n \rightarrow \infty} \rho\left(t_{n}, t_{n+1}\right)=0$,

then the set of all limit points of $t_{n}$ is connected. Next we use the expression

$$
\begin{aligned}
\int_{0}^{1}(g(x)-\tilde{g}(x))^{2} \mathrm{~d} x= & \int_{0}^{1} \int_{0}^{1}|x-y| \mathrm{d} g(x) \mathrm{d} \tilde{g}(y) \\
& -\frac{1}{2} \int_{0}^{1} \int_{0}^{1}|x-y| \mathrm{d} g(x) \mathrm{d} g(y)-\frac{1}{2} \iint_{0}^{1}|x-y| \mathrm{d} \tilde{g}(x) \mathrm{d} \tilde{g}(y) .
\end{aligned}
$$

Putting $g(x)=F\left(X_{n+1}, x\right)$ and $\tilde{g}(x)=F\left(X_{n}, x\right)$ we get the desired limit 3

As a consequence we have:

TheOREM 15. If $\lim _{n \rightarrow \infty} \frac{x_{n}}{x_{n+1}}=1$, then $G\left(X_{n}\right)$ is connected.

${ }^{3} \rho^{2}(g, \tilde{g})=\int_{0}^{1}(g(x)-\tilde{g}(x))^{2} \mathrm{~d} x$.

In Example 4 is given $X_{n}$ such that $G\left(X_{n}\right)$ is connected but $\lim _{\sup _{n \rightarrow \infty}} \rho\left(t_{n+1}, t_{n}\right)=1$. 
Pr o of. After some manipulation (17) it follows from

$$
\lim _{n \rightarrow \infty}\left(\frac{1}{n x_{n}} \sum_{i=1}^{n} x_{i}\right)\left(1-\frac{x_{n}}{x_{n+1}}\right)=0 .
$$

Note that by [24, Th. 4.1] all d.f.'s in $G\left(X_{n}\right)$ are continuous everywhere on $[0,1]$ if they are continuous at 0 and 1 .

In [24, Th. 3.2] is proved that if $g(x) \in G\left(X_{n}\right), g(x)$ increases at $\beta \in[0,1)$, $g(\beta)>0$, then there exists $\alpha \in[1, \infty)$ such that $\alpha g(x \beta) \in G\left(X_{n}\right)$. Using this fact, we can define on $G\left(X_{n}\right)$ the relation $\tilde{g}(x) \prec g(x)$ if there exist $\alpha, \beta$ such that $\tilde{g}(x)=\alpha g(x \beta)$. For every element $g(x) \in G\left(X_{n}\right)$ we define $[g(x)]$ as the set of all $\tilde{g}(x) \in G\left(X_{n}\right)$ for which $\tilde{g}(x) \prec g(x)$. Assuming that all d.f.s in $G\left(X_{n}\right)$ are continuous and strictly increasing, then we have

$$
[g(x)]=\{g(x \beta) / g(\beta) ; \beta \in(0,1]\} .
$$

Denote as $G(g(x))$ the set of all possible $\operatorname{limits}_{k \rightarrow \infty} g\left(x \beta_{k}\right) / g\left(\beta_{k}\right)$, where $\beta_{k} \rightarrow 0$ and put

$$
[g(x)]^{*}=[g(x)] \cup G(g(x)) .
$$

TheOREM 16. Assume that all d.f.s in $G\left(X_{n}\right)$ are continuous and strictly increasing. If $G\left(X_{n}\right)=\cup_{i=1}^{k}\left[g_{i}(x)\right]^{*}$, then $G\left(X_{n}\right)$ is connected if and only if $g_{i}(x)$, $i=1,2, \ldots, k$ can be reordered into $g_{i_{n}}(x), n=1,2, \ldots, k$ such that

(i) $\left[g_{i_{n}}(x)\right]^{*} \cap\left[g_{i_{n+1}}(x)\right]^{*} \neq \emptyset, n=1,2, \ldots, k-1$.

Proof. $1^{0}$ Firstly we prove that $[g(x)]^{*}$ is nonempty, closed and connected, for every $g(x) \in G\left(X_{n}\right)$. Note that, in the following we say that we can go connectively $g_{1}(x) \rightarrow g_{2}(x)$ through the set $H$ if for every $\varepsilon>0$ there exists a chain $g_{i_{n}}(x) \in H, n=1,2, \ldots, m$ such that $\rho\left(g_{1}, g_{i_{1}}\right)<\varepsilon, \rho\left(g_{i_{2}}, g_{i_{3}}\right)<\varepsilon, \ldots$ $\ldots, \rho\left(g_{i_{m}}, g_{2}\right)<\varepsilon$.

Connectivity: If $g_{1}(x)=g\left(x \beta_{1}\right) / g\left(\beta_{1}\right)$ and $g_{2}(x)=g\left(x \beta_{2}\right) / g\left(\beta_{2}\right)$ then we can go connectively $g_{1}(x) \rightarrow g_{2}(x)$ through $g(x \beta) / g(\beta)$, where $\beta$ is between $\beta_{1}$ and $\beta_{2}$, since

$$
\frac{g(x \beta)}{g(\beta)}-\frac{g\left(x \beta^{\prime}\right)}{g\left(\beta^{\prime}\right)}=\left(\frac{g(x \beta)-g\left(x \beta^{\prime}\right)}{g(\beta)}+g\left(x \beta^{\prime}\right) \frac{g\left(\beta^{\prime}\right)-g(\beta)}{g(\beta) g\left(\beta^{\prime}\right)}\right) \rightarrow 0
$$

as $\left(\beta^{\prime}-\beta\right) \rightarrow 0$, where $\beta, \beta^{\prime} \geq \varepsilon>0$.

If $g_{1}(x)=\lim _{k \rightarrow \infty} g\left(x \beta_{k}\right) / g\left(\beta_{k}\right)$ and $g_{2}(x)=\lim _{k \rightarrow \infty} g\left(x \beta_{k}^{\prime}\right) / g\left(\beta_{k}^{\prime}\right)$, then we can go connectively

$$
g_{1}(x) \rightarrow g\left(x \beta_{k}\right) / g\left(\beta_{k}\right) \rightarrow g\left(x \beta_{k}^{\prime}\right) / g\left(\beta_{k}^{\prime}\right) \rightarrow g_{2}(x)
$$

through $[g(x)]$. Similarly for the rest

$$
g_{1}(x)=g\left(x \beta_{1}\right) / g\left(\beta_{1}\right) \quad \text { and } \quad g_{2}(x)=\lim _{k \rightarrow \infty} g\left(x \beta_{k}\right) / g\left(\beta_{k}\right) \text {. }
$$




\section{DISTRIBUTION FUNCTIONS OF RATIO SEQUENCES. AN EXPOSITORY PAPER}

Closedness: If $\lim _{k \rightarrow \infty} g\left(x \beta_{k}\right) / g\left(\beta_{k}\right)=g_{1}(x)$, we can select $\beta_{k}$ such that $\beta_{k} \rightarrow \beta$. If $\beta>0$, then from continuity $g(x)$ we have $g_{1}(x)=g(x \beta) / g(\beta)$. The closedness of $G(g(x))$ follows from definition of $G(g(x))$.

$2^{0}$. Assume that (i) holds and select $g_{n}^{*}(x) \in\left[g_{i_{n}}(x)\right]^{*} \cap\left[g_{i_{n+1}}(x)\right]^{*}, i=1,2, \ldots$ $\ldots, k-1$. Let $g_{1}(x) \in\left[g_{i_{1}}(x)\right]^{*}$ and $g_{2}(x) \in\left[g_{i_{3}}(x)\right]^{*}$. Then we can go connectively

$$
g_{1}(x) \rightarrow \frac{g_{i_{1}}\left(x \beta_{1}\right)}{g_{i_{1}}\left(\beta_{1}\right)} \rightarrow g_{1}^{*}(x) \rightarrow \frac{g_{i_{2}}\left(x \beta_{2}\right)}{g_{i_{2}}\left(\beta_{2}\right)} \rightarrow g_{2}^{*}(x) \rightarrow \frac{g_{i_{3}}\left(x \beta_{3}\right)}{g_{i_{3}}\left(\beta_{3}\right)} \rightarrow g_{2}(x),
$$

similarly in a general case.

$3^{0}$. Assume that (i) does not hold. Then $\left[g_{i}(x)\right]^{*}, i=1,2, \ldots, k$, can be divided into two parts such that

$$
\left(\cup_{i \in A}\left[g_{i}(x)\right]^{*}\right) \cap\left(\cup_{i \in B}\left[g_{i}(x)\right]^{*}\right)=\emptyset,
$$

where $A \cup B=\{1,2, \ldots, k\}$. From closedness of such sets follows $\rho(g, \tilde{g}) \geq \delta>0$ for some $\delta$ and every $g(x) \in \cup_{i \in A}\left[g_{i}(x)\right]^{*}$ and $\tilde{g}(x) \in \cup_{i \in B}\left[g_{i}(x)\right]^{*}$, which contradicts the connectivity of $G\left(X_{n}\right)$.

4.7. Boundaries of $g(x) \in G\left(X_{n}\right)$

Theorem 17 ([3, Th. 5]). For every increasing sequence of positive integers $x_{n}$, $n=1,2, \ldots$, there exists $g(x) \in G\left(X_{n}\right)$ such that $g(x) \geq x$ for all $x \in[0,1]$.

P r o of. If $\underline{d}>0$, select $n_{k}$ so that $\frac{n_{k}}{x_{n_{k}}} \rightarrow \underline{d}>0$, and $F\left(X_{n_{k}}, x\right) \rightarrow g(x)$. For such $g(x)$, (5) implies

$$
\frac{g(x)}{x} \underline{d} \geq \underline{d} .
$$

Now, let $\underline{d}=0$. Select $n_{k}$ such that

$$
\frac{n_{k}}{x_{n_{k}}}=\min _{i \leq n_{k}} \frac{i}{x_{i}}
$$

and $F\left(X_{n_{k}}, x\right) \rightarrow g(x)$. Then for every $x \in(0,1]$,

$$
\frac{A\left(x x_{n_{k}}\right)}{x x_{n_{k}}} \geq \frac{n_{k}-1}{x_{n_{k}}} .
$$

Applying (2) yields

$$
\frac{F\left(X_{n_{k}}, x\right)}{x} \frac{n_{k}}{x_{n_{k}}} \geq \frac{n_{k}-1}{x_{n_{k}}},
$$

and taking the limit, as $k \rightarrow \infty$, we obtain $g(x) \geq x$ for all $x \in[0,1]$ [

${ }^{4}$ L. Mišík. 


\section{OTO STRAUCH}

Theorem 18 ([3, Th. 6]). Let $x_{1}<x_{2}<\ldots$ be a sequence of positive integers with positive lower asymptotic density $\underline{d}>0$, and upper asymptotic density $\bar{d}$. Then all d.f.s $g(x) \in G\left(X_{n}\right)$ are continuous, non-singular, and bounded by $h_{1}(x) \leq g(x) \leq h_{2}(x)$, where

$$
\begin{aligned}
& h_{1}(x)= \begin{cases}x \underline{\underline{\underline{d}}}, & \text { if } x \in\left[0, \frac{1-\bar{d}}{1-\underline{d}}\right] ; \\
\frac{\underline{d}}{x-(1-\underline{d})}, & \text { otherwise, }\end{cases} \\
& h_{2}(x)=\min \left(x \frac{\bar{d}}{\underline{d}}, 1\right) .
\end{aligned}
$$

Moreover, $h_{1}(x)$ and $h_{2}(x)$ are the best possible in the following sense: for given $0<\underline{d} \leq \bar{d}$, there exists $x_{1}<x_{2}<\cdots$ with lower and upper asymptotic densities $\underline{d}, \bar{d}$, such that $\underline{g}(x)=h_{1}(x)$ for $x \in\left[\frac{1-\bar{d}}{1-\underline{d}}, 1\right]$; also, there exists $x_{1}<x_{2}<\cdots$ with given $0<\underline{d} \leq \bar{d}$ such that $\bar{g}(x)=h_{2}(x) \in G\left(X_{n}\right)$.

Proof. For $g(x) \in G\left(X_{n}\right)$, let $n_{k}, k=1,2, \ldots$, be an increasing sequence of indices such that $F\left(X_{n_{k}}, x\right) \rightarrow g(x)$. From $n_{k}$ we can select a subsequence (for simplicity written as the original $n_{k}$ ) 5 such that

$$
\frac{n_{k}}{x_{n_{k}}} \rightarrow d_{g}>0
$$

Then, by (5), we have

$$
g(x)=x \frac{d_{g}(x)}{d_{g}}, \quad \text { where } \quad \frac{A\left(x x_{n_{k}}\right)}{x x_{n_{k}}} \rightarrow d_{g}(x)
$$

for arbitrary $x \in(0,1]$.

We will continue in six steps $1^{0}-6^{0}$.

$1^{0}$. We prove the continuity of $g(x)$ at $x=1$ (improving (iv) in [24, Th. 6.2]) for each $g(x) \in G\left(X_{n}\right)$.

In view of the definition of the counting function $A(t)$

$$
0 \leq A\left(x_{n_{k}}\right)-A\left(x x_{n_{k}}\right) \leq x_{n_{k}}-x x_{n_{k}} ;
$$

thus,

$$
0 \leq \frac{A\left(x_{n_{k}}\right)}{x_{n_{k}}}-\frac{A\left(x x_{n_{k}}\right)}{x_{n_{k}}}=\frac{n_{k}-1}{x_{n_{k}}}-\frac{A\left(x x_{n_{k}}\right)}{x x_{n_{k}}} x \leq 1-x,
$$

and, as $k \rightarrow \infty$, we have $0 \leq d_{g}-d_{g}(x) x \leq 1-x$, which implies

$$
0 \leq d_{g}-d_{g}(x)+d_{g}(x)(1-x) \leq 1-x .
$$

Consequently, $\lim _{x \rightarrow 1} d_{g}(x)=d_{g}$, and so $\lim _{x \rightarrow 1} g(x)=\lim _{x \rightarrow 1} x \frac{d_{g}(x)}{d_{g}}=1$. Since $g(x) \in G\left(X_{n}\right)$ is arbitrary, [24, Th. 4.1, Th. 6.2] gives continuity of $g(x)$ in the whole unit interval $[0,1]$.

${ }^{5}$ We call $d_{g}$ a local asymptotic density related to $g(x)$. 


\section{DISTRIBUTION FUNCTIONS OF RATIO SEQUENCES. AN EXPOSITORY PAPER}

$2^{0}$. We prove that $g(x)$ has a bounded right derivative for every $x \in(0,1)$, and for each $g(x) \in G\left(X_{n}\right)$.

For $0<x<y<1$ again

$$
0 \leq A\left(y x_{n_{k}}\right)-A\left(x x_{n_{k}}\right) \leq(y-x) x_{n_{k}},
$$

which implies

$$
0 \leq \frac{A\left(y x_{n_{k}}\right)}{y x_{n_{k}}} y-\frac{A\left(x x_{n_{k}}\right)}{x x_{n_{k}}} x \leq y-x .
$$

Letting $k \rightarrow \infty$, we get

$$
0 \leq d_{g}(y) y-d_{g}(x) x \leq y-x
$$

hence

Consequently,

$$
0 \leq g(y)-g(x)=\frac{d_{g}(y) y-d_{g}(x) x}{d_{g}} \leq \frac{y-x}{d_{g}}
$$

$$
0 \leq \frac{g(y)-g(x)}{y-x} \leq \frac{1}{d_{g}}
$$

for all $x, y \in(0,1), x<y$, which gives the upper bound of the right derivatives of $g(x)$ for every $x \in(0,1)$. Note that a singular d.f. (continuous, strictly increasing, having zero derivative a.e.) has infinite right Dini derivatives in a dense subset of $(0,1)$.

$3^{0}$. We prove a local form of Theorem 17.

As $\underline{d} \leq d_{g} \leq \bar{d}$, (21) implies

$$
x \frac{d}{d_{g}} \leq g(x) \leq x \frac{\bar{d}}{d_{g}}
$$

for every $x \in[0,1]$. It follows from (22), that there exists an extreme point $A_{g}=\left(x_{g}, y_{g}\right)$ on the line $y=x \frac{d}{d_{g}}$ such that $g(x)$ has no common point with this line for $x>x_{g}$. This point $A_{g}$ is the intersection of the lines

$$
y=x \frac{\underline{d}}{d_{g}} \quad \text { and }, \quad y=x \frac{1}{d_{g}}+1-\frac{1}{d_{g}}
$$

therefore,

$$
A_{g}=\left(x_{g}, y_{g}\right)=\left(\frac{1-d_{g}}{1-\underline{d}}, \frac{\underline{d}}{d_{g}} \frac{1-d_{g}}{1-\underline{d}}\right) .
$$

It means that for a given $g(x) \in G\left(X_{n}\right), h_{1, g}(x) \leq g(x) \leq h_{2, g}(x)$, where

$$
\begin{aligned}
& h_{1, g}(x)= \begin{cases}x \frac{d}{d_{g}}, & \text { if } x<y_{0}=\frac{1-d_{g}}{1-\underline{d}} \\
x \frac{1}{d_{g}}+1-\frac{1}{d_{g}}, & \text { if } y_{0} \leq x \leq 1,\end{cases} \\
& h_{2, g}(x)=\min \left(x \frac{\bar{d}}{d_{g}}, 1\right) .
\end{aligned}
$$




\section{OTO STRAUCH}

$4^{0}$. Now we find $h_{1}(x)$, and $h_{2}(x)$ such that

$$
h_{1}(x) \leq h_{1, g}(x) \leq h_{2, g}(x) \leq h_{2}(x)
$$

for every $g \in G\left(X_{n}\right)$.

In the parametric expression (25) of $A_{g}$, the local asymptotic density $d_{g}$ defined by $(20)$ belongs to the interval $[\underline{d}, \bar{d}]$. The well-known Darboux property of the asymptotic density implies that for an arbitrary $d \in[\underline{d}, \bar{d}]$ there exists an increasing $n_{k}, k=1,2, \ldots$, such that $\frac{n_{k}}{x_{n_{k}}} \rightarrow \sqrt{6}$, and then the Helly selection principle implies the existence of a subsequence of $n_{k}$ such that $F\left(X_{n_{k}}, x\right) \rightarrow g(x)$ for some $g(x) \in G\left(X_{n}\right)$. Thus, if $g(x)$ runs over $G\left(X_{n}\right)$, then $d_{g}$ runs over the entire interval $[\underline{d}, \bar{d}]$. Substituting $d_{g}=1-x_{g}(1-\underline{d})$ in $A_{g}=\left(x_{g}, y_{g}\right)$ we get

$$
y_{g}=y_{g}\left(x_{g}\right)=\frac{\underline{d}}{\frac{1}{x_{g}}-(1-\underline{d})},
$$

where $x_{g}=\frac{1-d_{g}}{1-\underline{d}}$ runs through the interval $I=\left[\frac{1-\bar{d}}{1-\underline{d}}, 1\right]$ for $d_{g} \in[\underline{d}, \bar{d}]$. By putting $x_{g}=x$, and $y_{g}=h_{1}$ we find a part of $h_{1}(x)$ for $\bar{x} \in I$ in (18). The remaining part of $h_{1}(x)$, and also the whole $h_{2}(x)$, follow from the basic inequality (23), see [3. Fig. 1.]. The optimality of $h_{1}(x)$ and $h_{2}(x)$ are proved in $5^{0}$ and $6^{0}$ pages $518-522$ of [3]. [7

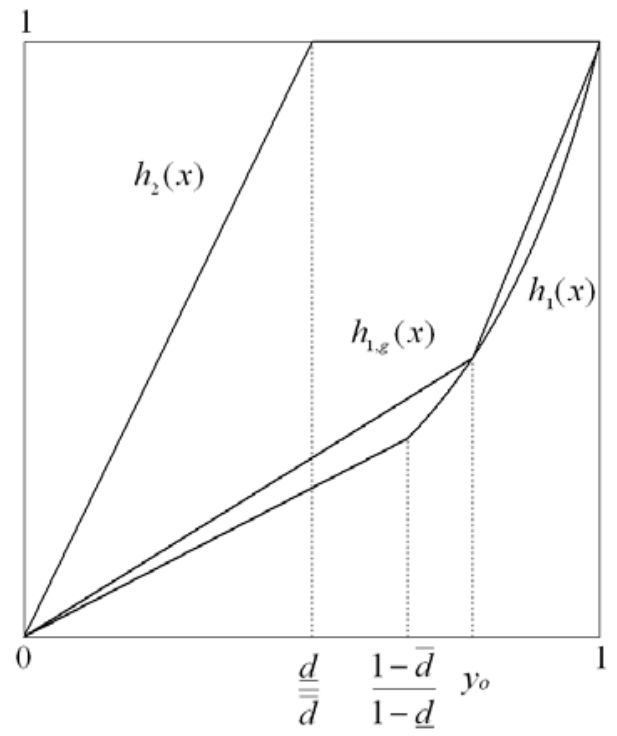

Figure: Boundaries of $g(x) \in G\left(X_{n}\right)$

\footnotetext{
${ }^{6}$ A simple proof follows from the fact that for every $d \in(\underline{d}, \bar{d})$ there exist infinitely many $n \in \mathbb{N}$ such that $A(n) / n \leq d \leq A(n+1) /(n+1)$. These $n$ we denote as $n_{k}$.

${ }^{7}$ L. Mišík for the idea of (22).
} 


\section{Application}

An application of d.f.s in Theorem 18 to elementary number theory:

Theorem 19 ([3, Th. 7]). For every increasing sequence $x_{1}<x_{2}<\cdots$ of positive integers with lower and upper asymptotic densities $0<\underline{d} \leq \bar{d}$ we have

$$
\begin{gathered}
\frac{1}{2} \frac{d}{\overline{\bar{d}}} \leq \liminf _{n \rightarrow \infty} \frac{1}{n} \sum_{i=1}^{n} \frac{x_{i}}{x_{n}} \\
\limsup _{n \rightarrow \infty} \frac{1}{n} \sum_{i=1}^{n} \frac{x_{i}}{x_{n}} \leq \frac{1}{2}+\frac{1}{2}\left(\frac{1-\min (\sqrt{\underline{d}}, \bar{d})}{1-\underline{d}}\right)\left(1-\frac{\underline{d}}{\min (\sqrt{d}, \bar{d})}\right) .
\end{gathered}
$$

Here the equations in (28) and (29) can be attained.

P r o of. By Helly theorem, if $F\left(X_{n_{k}}, x\right) \rightarrow g(x)$, then

$$
\int_{0}^{1} x \mathrm{~d} F\left(X_{n_{k}}, x\right)=\frac{1}{n_{k}} \sum_{i=1}^{n_{k}} \frac{x_{i}}{x_{n_{k}}} \rightarrow \int_{0}^{1} x \mathrm{~d} g(x)=1-\int_{0}^{1} g(x) \mathrm{d} x .
$$

If $\underline{d}>0$, then $h_{1}(x) \leq g(x) \leq h_{2}(x)$ which implies

$$
1-\int_{0}^{1} h_{2}(x) \mathrm{d} x \leq 1-\int_{0}^{1} g(x) \mathrm{d} x \leq 1-\int_{0}^{1} h_{1}(x) \mathrm{d} x .
$$

For $x_{1}<x_{2}<\cdots$ for which $h_{2}(x) \in G\left(X_{n}\right)$ in the left of (30) we have equation, but in every case $h_{1}(x) \notin G\left(X_{n}\right)$ for $0<\underline{d}<\bar{d}$, which implies strong inequality in the right, i.e.,

$$
\limsup _{n \rightarrow \infty} \frac{1}{n} \sum_{i=1}^{n} \frac{x_{i}}{x_{n}}<1-\frac{1}{2} \frac{\underline{\bar{d}}}{\overline{\bar{d}}}\left(\frac{1-\overline{\bar{d}}}{1-\underline{d}}\right)^{2}-\frac{\underline{d}}{(1-\underline{d})^{2}}\left(\log \frac{\underline{\bar{d}}}{\overline{\bar{d}}}-(\bar{d}-\underline{d})\right) .
$$

Since for every $g(x) \in G\left(X_{n}\right)$ in $3^{0}$ we have $h_{1, g}(x) \leq g(x) \leq h_{2, g}(x)$, then

$$
\limsup _{n \rightarrow \infty} \frac{1}{n} \sum_{i=1}^{n} \frac{x_{i}}{x_{n}} \leq \max _{g(x) \in G\left(X_{n}\right)}\left(1-\int_{0}^{1} h_{1, g}(x) \mathrm{d} x\right) .
$$

If the maximum in (32) is attained in $g_{0}(x) \in G\left(X_{n}\right)$ and $h_{1, g_{0}}(x) \in G\left(X_{n}\right)$, then $g_{0}(x)=h_{1, g_{0}}(x)$ and we have

$$
\limsup _{n \rightarrow \infty} \frac{1}{n} \sum_{i=1}^{n} \frac{x_{i}}{x_{n}}=1-\int_{0}^{1} h_{1, g_{0}}(x) \mathrm{d} x .
$$




\section{OTO STRAUCH}

Using (26) we find

$$
\int_{0}^{1} h_{1, g}(x) \mathrm{d} x=\frac{1}{2}\left(1+\frac{1-d_{g}}{1-\underline{d}}\left(\frac{\underline{d}}{d_{g}}-1\right)\right)
$$

for $d_{g} \in[\underline{d}, \bar{d}]$ with derivative $\left(\int_{0}^{1} h_{1, g}(x) \mathrm{d} x\right)^{\prime}=\frac{1}{2(1-\underline{d})}\left(1-\frac{\underline{d}}{\left(d_{g}\right)^{2}}\right)$ and which gives that $\min \int_{0}^{1} h_{1, g}(x) \mathrm{d} x$ is attained in $d_{g_{0}}=\min (\sqrt{\underline{d}}, \bar{d})$.

Now, to prove (33) we can construct integer $x_{1}<x_{2}<\cdots$ with $0<\underline{d} \leq \bar{d}$ such that $h_{1, g_{0}}(x) \in G\left(X_{n}\right)$.

We starting with the sequence of indices $n_{k}$, and then by (26) we must find indices $m_{k}^{\prime}<m_{k}<n_{k}$ and integers $x_{m_{k}^{\prime}}<x_{m_{k}}<x_{n_{k}}$ such that

(i) $\frac{n_{k}}{x_{n_{k}}} \rightarrow d_{g_{0}}$,

(ii) $\frac{m_{k}}{n_{k}} \rightarrow \frac{\underline{d}}{d_{g_{0}}} \frac{1-d_{g_{0}}}{1-\underline{d}}$,

(iii) $\frac{x_{m_{k}}}{x_{n_{k}}} \rightarrow \frac{1-d_{g_{0}}}{1-\underline{d}}$,

(iv) $\frac{x_{m_{k}^{\prime}}}{x_{n_{k}}} \rightarrow 0$

(v) $\frac{m_{k}^{\prime}}{n_{k}^{\prime}} \rightarrow 0$,

(vi) $\frac{m_{k}^{\prime}}{x_{m_{k}^{\prime}}} \rightarrow \bar{d}$.

Then from (i), (ii) and (iii) follows $\frac{m_{k}}{x_{m_{k}}} \rightarrow \underline{d}$. Furthermore we must again assumed

(v) $x_{m_{k}}-x_{m_{k}^{\prime}} \geq m_{k}-m_{k}^{\prime}$,

(vi) $x_{n_{k}}-x_{m_{k}} \geq n_{k}-m_{k}$,

(vii) $x_{m_{k+1}^{\prime}}-x_{n_{k}} \geq m_{k+1}^{\prime}-n_{k}$,

(viii) $n_{k}<m_{k+1}^{\prime}$,

(ix) $m_{1}^{\prime} \leq x_{m_{1}^{\prime}}$.

It can be solved naturally and complement values $x_{n}$ are defined linearly.

\section{Algorithm [4, p. 5]}

Let $1 \leq x_{1}<x_{2}<\cdots$ be an increasing sequence of positive integers. Put $x_{0}=0$ and

$$
t_{n}=x_{n}-x_{n-1}, \quad n=1,2, \ldots
$$

For every $n=1,2, \ldots$ we compute the finite integer sequence

$$
t_{1}^{(n)}, t_{2}^{(n)}, \ldots, t_{n}^{(n)}
$$

from $t_{1}, t_{2}, \ldots$ by the following procedure: 


\section{DISTRIBUTION FUNCTIONS OF RATIO SEQUENCES. AN EXPOSITORY PAPER}

$1^{0}$. For $n=1, t_{1}^{(1)}=t_{1}=x_{1}$;

$2^{0}$. For $n=2, t_{1}^{(2)}=t_{1}+t_{2}-1=x_{2}-1$ and $t_{2}^{(2)}=1$;

$3^{0}$. Assume that for $n-1 \geq 2$ we have $t_{i}^{(n-1)}, i=1,2, \ldots, n-1$. For $n$ we first define the initial auxiliary sequence $t_{1}^{\prime}, t_{2}^{\prime}, \ldots, t_{n}^{\prime}$ such that $t_{i}^{\prime}=t_{i}^{(n-1)}, i=1,2, \ldots, n-1$, and $t_{n}^{\prime}=t_{n}$. Then we repeatedly modify this sequence using following steps (a) and $(b)$.

(a) If there exists $k, 1<k<n$, such that $t_{1}^{\prime}=t_{2}^{\prime}=\cdots=t_{k-1}^{\prime}>t_{k}^{\prime}$ and $t_{n}^{\prime}>1$, then we put $t_{k}^{\prime}:=t_{k}^{\prime}+1, t_{n}^{\prime}:=t_{n}^{\prime}-1$ and $t_{i}^{\prime}:=t_{i}^{\prime}$ in all other cases.

(b) If such $k$ does not exist and $t_{n}^{\prime}>1$, then we put $t_{1}^{\prime}:=t_{1}^{\prime}+1, t_{n}^{\prime}:=t_{n}^{\prime}-1$ and $t_{i}^{\prime}:=t_{i}^{\prime}$ in all other cases.

Repeated application of (a) and (b) shows that the step $3^{0}$ terminates if $t_{n}^{\prime}=1$ and outputs the sequence $t_{1}^{(n)}:=t_{1}^{\prime}, \ldots, t_{n}^{(n)}:=t_{n}^{\prime}$.

$4^{0}$. Put $n-1:=n$ and use the output $t_{1}^{(n)}, \ldots, t_{n}^{(n)}$ as the new input in $3^{0}$.

Thus the final output of Algorithm is the infinite sequence of finite integers block $t_{1}^{(n)}, t_{2}^{(n)}, \ldots, t_{n}^{(n)}$ for $n=1,2, \ldots$

Lemma 1 ([4, Lemma 1]). Assuming that $t_{n} \neq 1$ for infinitely many $n$, then the output $t_{1}^{(n)}, t_{2}^{(n)}, \ldots, t_{n}^{(n)}$ of the Algorithm can be of the following two possible forms:

(A) $t_{1}^{(n)}=\cdots=t_{m}^{(n)}=D_{n}>t_{m+1}^{(n)} \geq t_{m+2}^{(n)}=t_{m+3}^{(n)}=\ldots t_{n}^{(n)}=1$,

(B) $t_{1}^{(n)}=\cdots=t_{m}^{(n)}=D_{n}>t_{m+1}^{(n)}=\cdots=t_{m+s}^{(n)}=D_{n}-1 \geq t_{m+s+1}^{(n)}=\cdots=t_{n}^{(n)}=1$, for some $m=m(n), s=s(n)$ and for $D_{n}:=t_{1}^{(n)}$.

Lemma 2 (4, Lemma 2]). For $D_{n}$ defined in Lemma[1 there are two possibilities:

(I) $D_{n}$ is bounded;

(II) $D_{n} \rightarrow \infty$.

In the case (I) we have only the form (A) and $D_{n}=$ const. $=c \geq 2$ for all sufficiently large $n$.

In the case (II) both cases (A) and (B) are possible.

\section{Construction [4, p. 8]}

Assume that, for every $n=1,2, \ldots$, we have given $n$-terms sequence

$$
t_{1}^{(n)}, t_{2}^{(n)}, \ldots, t_{n}^{(n)}
$$

such that for every $n=1,2, \ldots$

$$
t_{1}^{(n)} \leq t_{1}^{(n+1)}, t_{2}^{(n)} \leq t_{2}^{(n+1)}, \ldots, t_{n}^{(n)} \leq t_{n}^{(n+1)} .
$$




\section{OTO STRAUCH}

Then, we define $x_{n}, x_{j}^{(n)}$ and $X_{n}^{(n)}$ as

$$
\begin{aligned}
x_{n} & =\sum_{i=1}^{n} t_{i}^{(n)}, \quad n=1,2, \ldots ; \\
x_{j}^{(n)} & =\sum_{i=1}^{j} t_{i}^{(n)}, \quad j=1,2, \ldots, n ; \\
X_{n}^{(n)} & =\left(\frac{x_{1}^{(n)}}{x_{n}^{(n)}}, \frac{x_{2}^{(n)}}{x_{n}^{(n)}}, \ldots, \frac{x_{n}^{(n)}}{x_{n}^{(n)}}\right), \quad n=1,2, \ldots
\end{aligned}
$$

Clearly $x_{n}^{(n)}=x_{n}$ and using (34) we see that

$$
x_{j}=\sum_{i=1}^{j} t_{i}^{(j)} \leq \sum_{i=1}^{j} t_{i}^{(n)}=x_{j}^{(n)}, \quad j=1,2, \ldots, n
$$

which implies

$$
F\left(X_{n}^{(n)}, x\right) \leq F\left(X_{n}, x\right) \quad \text { for all } \quad x \in[0,1], \quad n=1,2, \ldots
$$

Selecting a sequence of indices $n_{k}, k=1,2, \ldots$, such that $F\left(X_{n_{k}}, x\right) \rightarrow g(x)$ and $F\left(X_{n_{k}}^{\left(n_{k}\right)}, x\right) \rightarrow \tilde{g}(x)$ for all $x \in[0,1]$, we have

$$
\tilde{g}(x) \leq g(x) \quad \text { for all } \quad x \in[0,1] .
$$

\section{The case $\underline{d}=0[4$, p. 12]}

In the case $\underline{d}=0$ the Algorithm implies $\lim _{n \rightarrow \infty} D_{n}=\infty$ since if $D_{n}=$ const. $=c$, then $t_{1}^{(n)}, t_{2}^{(n)}, \ldots, t_{n}^{(n)}$ satisfy $(\mathrm{A})$ and $d_{g}=\frac{1}{\alpha(c-1)+1} \geq \frac{1}{c}>0$. Note that, in the opposite direction, $\lim _{n \rightarrow \infty} D_{n}=\infty$ need not imply $\underline{d}=0$, see the Construction.

The following theorem we shall formulate for the case (B), since the case (A) gives the same result, putting $\gamma=0$ and $s_{k}=0$.

Theorem 20 (4, Th. 3]). Let $x_{n}, n=1,2, \ldots$, be an increasing sequence of positive integers such that $\underline{d}=0$ and let $t_{1}^{(n)}, t_{2}^{(n)}, \ldots, t_{n}^{(n)}$ be a sequence produced by Algorithm. For a selected sequence of indices $n_{k}, k=1,2, \ldots$, assume that

(i) $F\left(X_{n_{k}}, x\right) \rightarrow g(x)$ and $F\left(X_{n_{k}}^{\left(n_{k}\right)}, x\right) \rightarrow \tilde{g}(x)$ for all $x \in[0,1]$;

(ii) $t_{1}^{\left(n_{k}\right)}=\cdots=t_{m_{k}}^{\left(n_{k}\right)}=D_{n_{k}}>t_{m_{k}+1}^{\left(n_{k}\right)}=\cdots=t_{m_{k}+s_{k}}^{\left(n_{k}\right)}=D_{n_{k}}-1$ $\geq t_{m_{k}+s_{k}+1}^{\left(n_{k}\right)}=\cdots=t_{n_{k}}^{\left(n_{k}\right)}=1$;

(iii) $\frac{m_{k}}{n_{k}} \rightarrow \alpha$;

(iv) $\frac{s_{k}}{n_{k}} \rightarrow \gamma$.

Then we have $\tilde{g}(x) \leq g(x)$ for all $x \in[0,1]$, where 
(a) If $\alpha+\gamma>0$ then $d_{g}=0$ and $\tilde{g}(x)=x(\alpha+\gamma)$ for all $x \in[0,1]$.

(b) If $\alpha+\gamma=0$ and $\frac{m_{k}+s_{k}}{n_{k}} D_{n_{k}} \rightarrow \infty$ then $d_{g}=0$ and $\tilde{g}(x)=0$ for all $x \in(0,1)$.

(c) If $\alpha+\gamma=0$ and $\frac{m_{k}+s_{k}}{n_{k}} D_{n_{k}} \rightarrow \delta, 0<\delta<\infty$, then $d_{g}=\frac{1}{\delta+1}$ and

$$
\tilde{g}(x)= \begin{cases}0 & \text { if } x<y_{2}=\frac{\delta}{\delta+1}, \\ x(\delta+1)-\delta & \text { if } y_{2} \leq x \leq 1\end{cases}
$$

(d) If $\alpha+\gamma=0$ and $\frac{m_{k}+s_{k}}{n_{k}} D_{n_{k}} \rightarrow \delta=0$, then $d_{g}=1$ and $\tilde{g}(x)=x$.

\subsection{Lower and upper d.f.s}

In Theorem 17 we gave the result [3, Th. 6] that for every integer sequence $1 \leq x_{1}<x_{2}<\cdots$ with $\underline{d}>0$ and every d.f. $g(x) \in G\left(X_{n}\right)$ we have $h_{1}(x) \leq$ $g(x) \leq h_{2}(x)$, where $h_{1}(x)$ and $h_{2}(x)$ are defined in (18) and (19), respectively. Furthermore, by [3, Th. $6,6^{0}$ of Proof], there exists an integer sequence $1 \leq$ $x_{1}<x_{2}<\cdots$ with $\underline{d}>0$ such that $h_{2}(x) \in G\left(X_{n}\right)$. In this case $h_{2}(x)=\bar{g}(x)$ and $G\left(X_{n}\right)$ has the following additional properties.

Theorem 21 ([4, Th. 5]). Let $1 \leq x_{1}<x_{2}<\cdots$ be an integer sequence with $\underline{d}>0$ such that $h_{2}(x) \in G\left(X_{n}\right)$. Then the set $G\left(X_{n}\right)$ contains uncountable many different d.f.s $g_{\alpha}(x), \alpha \in[1, \infty)$, of the form

$$
g_{\alpha}(x)= \begin{cases}x \frac{1}{\alpha \beta} \underline{\frac{d}{d}} & \text { if } x \in\left[0, \frac{d}{\overline{\bar{d}}} \beta\right], \\ \frac{1}{\alpha} & \text { if } x \in\left[\frac{\underline{\underline{d}}}{\bar{d}} \beta, \beta\right], \\ \text { nondecreasing } & \text { if } x \in[\beta, 1],\end{cases}
$$

where for $\beta=\beta(\alpha)$ we have $1 \leq \alpha \beta \leq \frac{\bar{d}}{\underline{d}}$. Furthermore, $g(x)=x$ is also in $G\left(X_{n}\right)$. P r o of. We use two steps.

$1^{0}$. Assume that $F\left(X_{n_{k}}, x\right) \rightarrow h_{2}(x)$ as $k \rightarrow \infty$ for $x \in[0,1]$. For every $\alpha \in[1, \infty)$ we can choose $n_{k}^{\prime}>n_{k}$ so that

(i) $\frac{n_{k}^{\prime}}{n_{k}} \rightarrow \alpha$.

From the sequence $\left(n_{k}^{\prime}, n_{k}\right), k=1,2, \ldots$, we can select a subsequence (with the same notation) such that

(ii) $\frac{x_{n_{k}}}{x_{n_{k}^{\prime}}} \rightarrow \beta$,

where $\beta=\beta(\alpha)$ but it is not given uniquely. We have only $\frac{1}{\alpha} \frac{\overline{\underline{\underline{d}}}}{\mathrm{~d}} \leq \beta \leq \frac{1}{\alpha} \frac{\bar{d}}{\underline{d}}$ because

$$
\frac{n_{k}^{\prime}}{n_{k}} \frac{x_{n_{k}}}{x_{n_{k}^{\prime}}}=\frac{\frac{n_{k}^{\prime}}{x_{n_{k}^{\prime}}}}{\frac{n_{k}}{x_{n_{k}}}} \rightarrow \alpha \beta
$$

and which gives $\alpha<\infty \Leftrightarrow \beta>0$. Now, from $\left(n_{k}^{\prime}, n_{k}\right)$ we again select a subsequence such that 


\section{OTO STRAUCH}

(iii) $F\left(X_{n_{k}^{\prime}}, x\right) \rightarrow g(x)$

for all $x \in[0,1]$. Applying the identity (1)

$$
F\left(X_{n_{k}}, x\right)=\frac{n_{k}^{\prime}}{n_{k}} F\left(X_{n_{k}^{\prime}}, x \frac{x_{n_{k}}}{x_{n_{k}^{\prime}}}\right)
$$

and assuming that $\underline{d}>0$, which implies everywhere continuity of $g(x)$ (see [24, Th. 6.2]) and $g(x)>0$ for $0<x \leq 1$, then we can take limit in (41) to obtain

$$
h_{2}(x)=\alpha g_{\alpha}(x \beta)
$$

for $x \in[0,1]$. Now, using $h_{2}(x)=1$ for $x \in\left[\frac{\underline{\underline{d}}}{d}, 1\right]$, (42) implies $g_{\alpha}(x)=\frac{1}{\alpha}$ for $x \in[\underline{\underline{\underline{d}}} \beta, \beta]$ and $h_{2}^{\prime}(x)=\frac{\bar{d}}{\underline{d}}$ for $x \in[0, \underline{\underline{\bar{d}}}]$ implies $g_{\alpha}^{\prime}(x)=\frac{\bar{d}}{\underline{d}} \frac{1}{\alpha \beta}$ for $x \in[0, \underline{\underline{\underline{d}}} \beta]$. Then we obtain (40) and since $g_{\alpha}(x) \leq h_{2}(x)$, then $1 \leq \alpha \beta$.

$2^{0}$. Again, let $F\left(X_{n_{k}}, x\right) \rightarrow h_{2}(x)$ for $x \in[0,1]$. For every limit point $8 \beta>0$ of $\frac{x_{i}}{x_{n_{k}}}, i=1,2, \ldots, n_{k}, k=1,2, \ldots$, we can select $m_{k}<n_{k}$ such that
(i) $\frac{x_{m_{k}}}{x_{n_{k}}} \rightarrow \beta$,
(ii) $\frac{n_{k}}{m_{k}} \rightarrow \alpha$
(iii) $F\left(X_{m_{k}}, x\right) \rightarrow g(x)$.

The identity (11) in the form $F\left(X_{m_{k}}, x\right)=\frac{n_{k}}{m_{k}} F\left(X_{n_{k}}, x \frac{x_{m_{k}}}{x_{n_{k}}}\right)$ implies

$$
g(x)=\alpha h_{2}(x \beta)=\frac{h_{2}(x \beta)}{h_{2}(\beta)}
$$

for $x \in[0,1]$. From the form of $h_{2}(x)$ we have guaranteed that $\beta \in\left[0, \frac{d}{\bar{d}}\right]$ is a limit point of $\frac{x_{i}}{x_{n_{k}}}$ and in this case (43) gives

$$
g(x)=\frac{x \beta \frac{\bar{d}}{\underline{d}}}{\beta \frac{\bar{d}}{\underline{d}}}=x .
$$

For $\beta>\frac{d}{\bar{d}}$, if exists, we have $g(x)=h_{2}(x \beta)$ for $x \in[0,1]$, i.e.,

$$
g(x)= \begin{cases}x \beta \frac{\bar{d}}{\underline{d}} & \text { if } x \in\left[0, \underline{\underline{\bar{d}}} \frac{1}{\beta}\right] \\ 1 & \text { if } x \in\left[\underline{\overline{\bar{d}}} \frac{1}{\beta}, 1\right]\end{cases}
$$

Finally, for $h_{2}(x)$ defined in (19) for which $h_{2}(x)=\bar{g}(x)$ for special $1 \leq x_{1}<$ $x_{2}<\cdots$, we see directly that

$$
h_{2}(x y) \leq h_{2}(x) h_{2}(y)
$$

\footnotetext{
${ }^{8}$ In the following $\alpha$ and $\beta$ have another meaning as in $1^{0}$.
} 


\section{DISTRIBUTION FUNCTIONS OF RATIO SEQUENCES. AN EXPOSITORY PAPER}

for every $x, y \in[0,1]$. Also for $h_{1}(x)$ defined in (18), in the case $x \geq \sqrt{\frac{1-\bar{d}}{1-\underline{d}}}$, for which there exists a special sequence $x_{n}$ (see [24, pp. 774-777, Ex. 11.2]) such that the lower d.f. $\underline{g}(x)=h_{1}(x)$ we have 9

$$
\left(\frac{\underline{d}}{\frac{1}{x}-(1-\underline{d})}\right)\left(\frac{\underline{d}}{\frac{1}{y}-(1-\underline{d})}\right) \leq \frac{\underline{d}}{\frac{1}{x y}-(1-\underline{d})}
$$

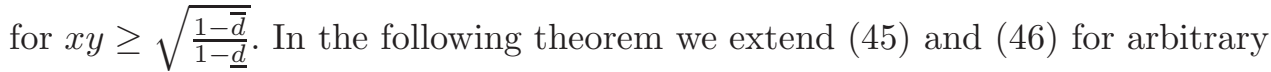
lower $\underline{g}(x)$ and upper $\bar{g}(x)$ d.f.s.

Theorem 22 ([4, Th. 6]). For every increasing sequence of positive integers $1 \leq x_{1}<x_{2}<\cdots$, with $\underline{d}>0$, the lower d.f. $\underline{g}(x)$ and the upper d.f. $\bar{g}(x)$ satisfy

$$
\underline{g}(x) \cdot \underline{g}(y) \leq \underline{g}(x \cdot y) \leq \bar{g}(x \cdot y) \leq \bar{g}(x) \cdot \bar{g}(y)
$$

for every $x, y \in(0,1)$.

Proof. $\underline{d}>0$ implies that arbitrary $g(x) \in G\left(X_{n}\right)$ is everywhere continuous and $g(x)>0$ for $x>0$. Let $y \in(0,1)$.

$1^{0}$. Firstly we prove the left-hand side of (47).

a) If $y$ is an increasing point 10 of $g(x), n=1,2, \ldots$ then by (6) we have $\frac{g(x y)}{g(y)} \in G\left(X_{n}\right)$ and thus $\underline{g}(x) \leq \frac{g(x y)}{g(y)}$ which implies

$$
\underline{g}(x) \underline{g}(y) \leq \underline{g}(x) g(y) \leq g(x y)
$$

for every $x \in(0,1)$.

b) Let $g(x)$ does not increase at $y$. Since every $g(x) \in G\left(X_{n}\right)$ is continuous and $\frac{d}{\bar{d}} x \leq g(x) \leq \frac{\bar{d}}{\underline{d}} x$ for $x \in[0,1]$, there exists the nearest neighboring point $y_{1}<y, y_{1}>0$ at which $g(x)$ increases. Thus $\frac{g\left(x y_{1}\right)}{g\left(y_{1}\right)} \in G\left(X_{n}\right)$ which implies $\underline{g}(x) \leq \frac{g\left(x y_{1}\right)}{g\left(y_{1}\right)}$. Because $g\left(y_{1}\right)=g(y), g\left(x y_{1}\right) \leq g(x y)$, then again

$$
\underline{g}(x) \underline{g}(y) \leq \underline{g}(x) g(y)=\underline{g}(x) g\left(y_{1}\right) \leq g\left(x y_{1}\right) \leq g(x y)
$$

for every $x \in(0,1)$.

Since $g \in G\left(X_{n}\right)$ is arbitrary, and for $x, y \in(0,1)$ by (47) and (48) we have $\underline{g}(x) \underline{g}(y) \leq g(x y)$, then the definition of lower d.f. of $G\left(X_{n}\right)$ as

$$
\underline{g}(x y)=\inf _{g \in G\left(X_{n}\right)} g(x y) \text { implies } \underline{g}(x) \underline{g}(y) \leq \underline{g}(x y) .
$$

$2^{0}$. Now, we prove the right-hand side of (47).

\footnotetext{
${ }^{9}$ This holds also for arbitrary $x, y \in(0,1)$, since it is equivalent to $x(1-y) \leq 1-y$.

${ }^{10}$ Either $g(y-\varepsilon)<g(y)$ or $g(y)<g(y+\varepsilon)$, for arbitrary $\varepsilon>0$.
} 


\section{OTO STRAUCH}

a) Again, if $y$ is an increasing point of $g(x)$, then $\frac{g(x y)}{g(y)} \in G\left(X_{n}\right)$, thus $\frac{g(x y)}{g(y)} \leq \bar{g}(x)$ which implies

$$
g(x y) \leq g(y) \bar{g}(x) \leq \bar{g}(y) \bar{g}(x)
$$

for $x \in(0,1)$.

b) Let $g(x)$ be non increasing at $y$ and let $y_{2}$ be the nearest point to the right at which $g(x)$ is increasing. Again, by $\frac{\underline{\underline{d}}}{d} x \leq g(x) \leq \frac{\bar{d}}{d} x$, this point exists and thus for given $g(x) \in G\left(X_{n}\right)$ we have $\frac{g\left(x y_{2}\right)}{g\left(y_{2}\right)} \in G\left(X_{n}\right), \frac{g\left(x y_{2}\right)}{g\left(y_{2}\right)} \leq \bar{g}(x)$ which implies

$$
g(x y) \leq g\left(x y_{2}\right) \leq g\left(y_{2}\right) \bar{g}(x) \leq g(y) \bar{g}(x) \leq \bar{g}(y) \bar{g}(x)
$$

for $x \in(0,1)$. Then

$$
\bar{g}(x y)=\sup _{g \in G\left(X_{n}\right)} g(x y) \text { implies } \bar{g}(x \cdot y) \leq \bar{g}(x) \cdot \bar{g}(y)
$$

for $x, y \in(0,1)$.

Note that by J. A c zél [1, p. 144-145, Th. 4] every continuous d.f. $g(x y)=$ $g(x) g(y)$ has the form $g(x)=x^{c}$ for a constant $c$ and $x \in[0,1]$.

\subsection{Construction $H \subset G\left(X_{n}\right)$}

Basic open problem is that characterize a nonempty set $H$ of d.f.s for which there exists an increasing sequence of positive integers $x_{n}$ such that $G\left(X_{n}\right)=H$. In [3] we found integer sequence $1 \leq x_{1}<x_{2}<\cdots$ such that the piecewise linear function $h_{2}(x)$ defined in (19) belongs to $G\left(X_{n}\right)$. In [4] is the following extension of this construction:

Theorem 23. Let $H$ be a nonempty set of d.f.s defined on $[0,1]$. Then there exists an integer sequence $1 \leq x_{1}<x_{2}<\cdots$ such that $H \subset G\left(X_{n}\right)$.

Proof.

$1^{0}$. To the set $H$ it can be constructed a sequence of continuous strictly increasing piecewise linear functions $h_{n}(x), n=1,2, \ldots$, such that every $f(x) \in H$ is a weak limit $h_{n_{k}}(x) \rightarrow f(x)$.

$2^{0}$. For every $h(x)$ possessing at points $\beta_{1}=0<\beta_{2}<\cdots<\beta_{s-1}<\beta_{s}=1$ the values $\alpha_{1}=0<\alpha_{2}<\cdots<\alpha_{s-1}<\alpha_{s}=1$, respectively, and being linear in each interval $\left[\beta_{i}, \beta_{i+1}\right]$, we can define a sequence of integer intervals $\left[m_{k}^{(1)}, n_{k}\right]$, $k=1,2, \ldots$, and their divisions

$$
m_{k}^{(1)}<m_{k}^{(2)}<\cdots<m_{k}^{(s-1)}<m_{k}^{(s)}<n_{k}
$$

in which we can define integers

$$
x_{m_{k}^{(1)}}<x_{m_{k}^{(2)}}<\cdots<x_{m_{k}^{(s-1)}}<x_{m_{k}^{(s)}}<x_{n_{k}}
$$

such that for $i=1,2, \ldots, s$ we have 
(i) $\frac{x_{m_{k}^{(i)}}}{x_{n_{k}}} \rightarrow \beta_{i}$,

(ii) $\frac{m_{k}^{(i)}}{n_{k}} \rightarrow \alpha_{i}$

(iii) $x_{m_{k}^{(i)}}-x_{m_{k}^{(i-1)}} \geq m_{k}^{(i)}-m_{k}^{(i-1)}$,

(iv) $x_{n_{k}}-x_{m_{k}^{(s)}} \geq n_{k}-m_{k}^{(s)}$.

For other $n \in\left[m_{k}^{(1)}, n_{k}\right]$ we define $x_{n}$ linearly, i.e., for $n \in\left[m_{k}^{(i-1)}, m_{k}^{(i)}\right]$ we put (v)

$$
x_{n}=x_{m_{k}^{(i-1)}}+\left[\left(n-m_{k}^{(i-1)}\right) \frac{x_{m_{k}^{(i)}}-x_{m_{k}^{(i-1)}}}{m_{k}^{(i)}-m_{k}^{(i-1)}}\right] .
$$

Directly from (i), (ii) and (v) it follows that

$$
\frac{\#\left\{n \in\left[m_{k}^{(1)}, n_{k}\right] ; \frac{x_{n}}{x_{n_{k}}}<x\right\}}{n_{k}} \rightarrow h(x) \text { for } x \in(0,1) \text { as } k \rightarrow \infty .
$$

See the following Fig. 1 and Fig. 2.

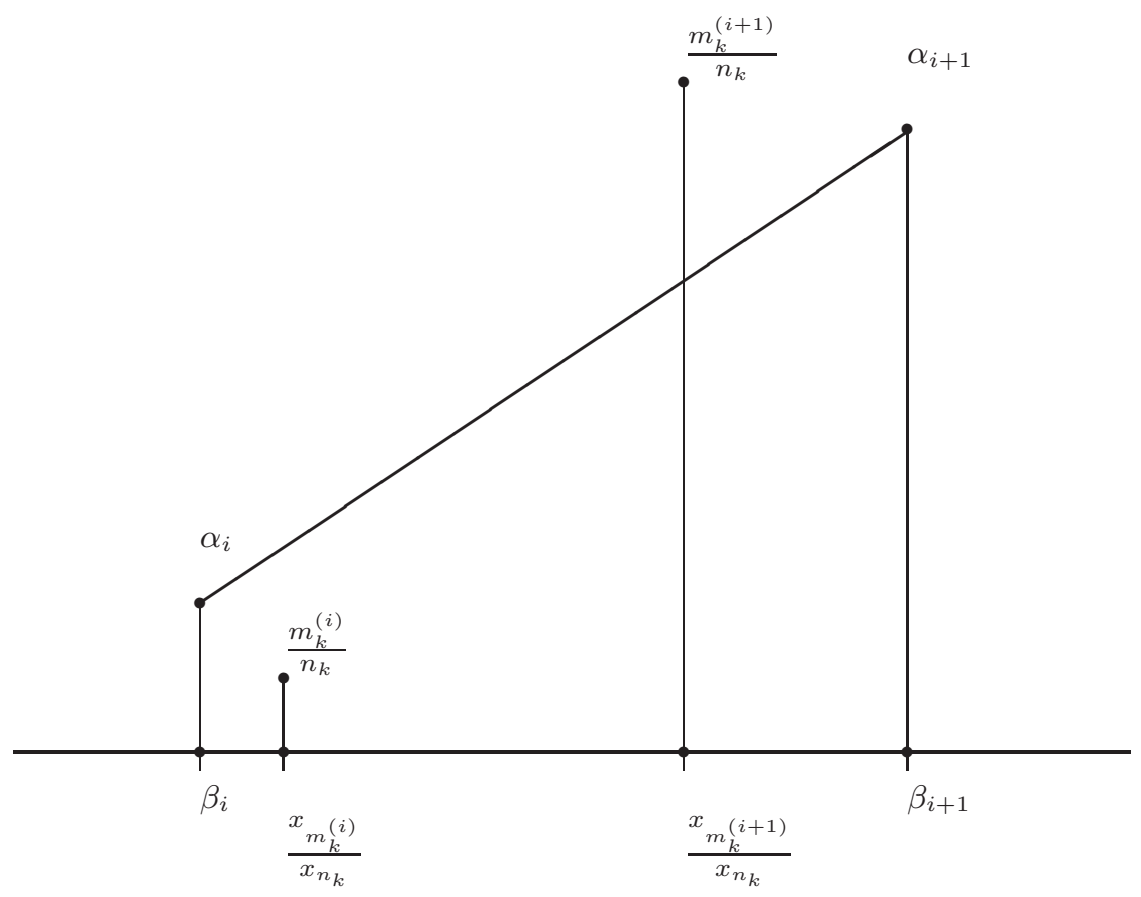

Figure 1. A part of graph of $h(x)$ and (i)-(ii) properties. 


\section{OTO STRAUCH}

Note that, in this step, the intervals $\left[m_{k}^{(1)}, n_{k}\right], k=1,2, \ldots$, can intersect. For necessity of pairwise disjointness we use the next step.
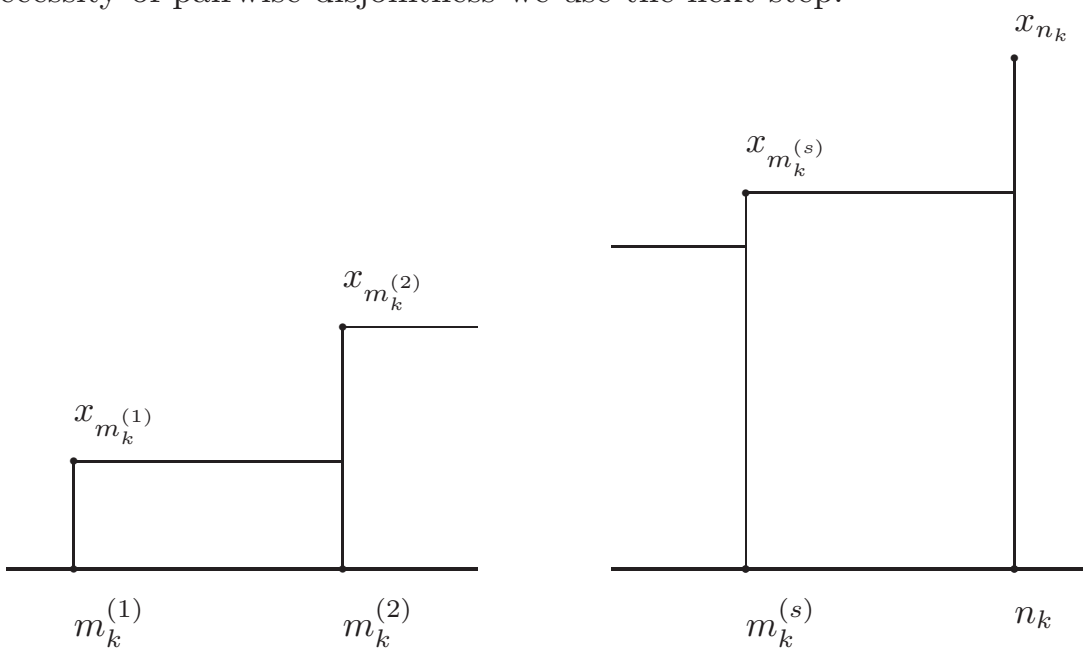

Figure 2. (iii)-(iv) properties.

$3^{0}$. One solution $\left[m_{k}^{(1)}, n_{k}\right], k=1,2, \ldots$ in $2^{0}$ gives infinitely many solutions by the following: Let $A_{k}<B_{k}$ be two positive integer sequences. Replace $\left[m_{k}^{(1)}, n_{k}\right]$ by $\left[A_{k} m_{k}^{(1)}, A_{k} n_{k}\right]$ with division

$$
A_{k} m_{k}^{(1)}<A_{k} m_{k}^{(2)}<\cdots<A_{k} m_{k}^{(s-1)}<A_{k} m_{k}^{(s)}<A_{k} n_{k}
$$

and define the values of $x_{n}$ as

$$
x_{A_{k} m_{k}^{(i)}}=B_{k} x_{m_{k}^{(i)}},
$$

$i=1,2, \ldots, s$ and $x_{A_{k} n_{k}}=B_{k} x_{n_{k}}$. Then the limits (i) and (ii) again hold

$$
\frac{x_{A_{k} m_{k}^{(i)}}}{x_{A_{k} n_{k}}}=\frac{B_{k} x_{m_{k}^{(i)}}}{B_{k} x_{n_{k}}} \rightarrow \beta_{i}, \quad \frac{A_{k} m_{k}^{(i)}}{A_{k} n_{k}} \rightarrow \alpha_{i} .
$$

Also (iii) and (iv) hold, since

$$
\begin{aligned}
x_{A_{k} m_{k}^{(i)}}-x_{A_{k} m_{k}^{(i-1)}} & =B_{k} x_{m_{k}^{(i)}}-B_{k} x_{m_{k}^{(i-1)}} \\
& \geq B_{k}\left(m_{k}^{(i)}-m_{k}^{(i-1)}\right) \geq A_{k} m_{k}^{(i)}-A_{k} m_{k}^{(i-1)} .
\end{aligned}
$$

$4^{0}$. Let $h_{i}(x), i=1,2, \ldots$ be a dense set of d.f.s in $H$ and for $h_{i}(x)=h(x)$ rewrite the interval $\left[m_{k}^{(1)}, n_{k}\right]$ in $2^{0}$ as $\left[m_{k}^{(1, i)}, n_{k}^{(i)}\right]$. Order these intervals to infinite 


\section{DISTRIBUTION FUNCTIONS OF RATIO SEQUENCES. AN EXPOSITORY PAPER}

$\operatorname{matrix} \mathbb{A}$

$$
\begin{aligned}
& {\left[m_{1}^{(1,1)}, n_{1}^{(1)}\right],\left[m_{2}^{(1,1)}, n_{2}^{(1)}\right], \ldots,\left[m_{k}^{(1,1)}, n_{k}^{(1)}\right], \ldots} \\
& {\left[m_{1}^{(1,2)}, n_{1}^{(2)}\right],\left[m_{2}^{(1,2)}, n_{2}^{(2)}\right], \ldots,\left[m_{k}^{(1,2)}, n_{k}^{(2)}\right], \ldots} \\
& \ldots \\
& {\left[m_{1}^{(1, i)}, n_{1}^{(i)}\right],\left[m_{2}^{(1, i)}, n_{2}^{(i)}\right], \ldots,\left[m_{k}^{(1, i)}, n_{k}^{(i)}\right], \ldots} \\
& \ldots
\end{aligned}
$$

and reorder it to a linear sequence by diagonals, i.e., to

$$
\left[m_{1}^{(1,1)}, n_{1}^{(1)}\right],\left[m_{1}^{(1,2)}, n_{1}^{(2)}\right],\left[m_{2}^{(1,1)}, n_{2}^{(1)}\right], \ldots
$$

and denote it as a new sequence $\left[m_{k}^{(1)}, n_{k}\right], k=1,2, \ldots$ Since these intervals can intersect we use in $3^{0}$ suitable $A_{k}<B_{k}, k=1,2, \ldots$ such that the resulting sequence is disjoint and

(vi) $x_{m_{k+1}^{(1)}}-x_{n_{k}} \geq m_{k+1}^{(1)}-n_{k}$,

(vii) $x_{m_{1}^{(1)}} \geq m_{1}^{(1)}$.

For $n$ which are not in the intervals $\left[m_{k}^{(1)}, n_{k}\right], k=1,2, \ldots$ we can define $x_{n}$ linearly. Now, if from $n_{k}, k=1,2, \ldots$ we select $n_{k}^{\prime}$ corresponding to $i$ th line of $\mathbb{A}$, then $F\left(X_{n_{k}^{\prime}}, x\right) \rightarrow h_{i}(x)$ for $x \in[0,1]$.

$5^{0}$. Finally, we give a solution of (i)-(iv) in $2^{0}$. We start with increasing sequence of indices $n_{k}, k=1,2, \ldots$, and let $\lambda>1$ and put (integer parts are omitted)

$$
\begin{aligned}
x_{n_{k}} & =\lambda n_{k}, \\
x_{m_{k}^{(i)}} & =\beta_{i} \lambda n_{k}, \\
m_{k}^{(i)} & =\alpha_{i} n_{k} .
\end{aligned}
$$

For (iv) we need

$$
\begin{aligned}
x_{m_{k}^{(i)}}-x_{m_{k}^{(i-1)}}=\beta_{i} \lambda n_{k}-\beta_{i-1} \lambda n_{k} & =\lambda\left(\beta_{i}-\beta_{i-1}\right) n_{k} \\
& \geq m_{k}^{(i)}-m_{k}^{(i-1)}=\left(\alpha_{i}-\alpha_{i-1}\right) n_{k}
\end{aligned}
$$

which gives assumption $\lambda>\max \frac{\alpha_{i}-\alpha_{i-1}}{\beta_{i}-\beta_{i-1}}$.

Note that by Theorem 23 there exists an integer sequence $1 \leq x_{1}<x_{2}<\cdots$ such that $G\left(X_{n}\right)$ contains all d.f.s. Especially, for every sequence $y_{n} \in[0,1)$, $n=1,2, \ldots$, there exists an $X_{n}$ such that $G\left(y_{n}\right) \subset G\left(X_{n}\right)$. 


\section{OTO STRAUCH}

\subsection{0. $g(x) \in G\left(X_{n}\right)$ with constant intervals}

Theorem $24(23])$. Assume that $\underline{d}>0$. If there exists an interval $(u, v) \subset[0,1]$ such that every $g \in G\left(X_{n}\right)$ has a constant value on $(u, v)$ (may be different), then every $g \in G\left(X_{n}\right)$ has infinitely many intervals with constant values such that $g$ increases at their endpoints.

Pro of. Since

$$
x_{i}<x x_{m} \Longleftrightarrow x_{i}<\left(x \frac{x_{m}}{x_{n}}\right) x_{n},
$$

then we have (1)

$$
F\left(X_{m}, x\right)=\frac{n}{m} F\left(X_{n}, x \frac{x_{m}}{x_{n}}\right),
$$

for every $m \leq n$ and $x \in[0,1)$. Using the Helly selection principle, we can select a subsequence $\left(m_{k}, n_{k}\right)$ of the sequence $(m, n)$ such that $F\left(X_{n_{k}}\right) \rightarrow g(x)$, $F\left(X_{m_{k}}\right) \rightarrow \tilde{g}(x)$ as $k \rightarrow \infty$; furthermore $x_{m_{k}} / x_{n_{k}} \rightarrow \beta$ and $n_{k} / m_{k} \rightarrow \alpha$, but $\alpha$ may be infinity. Assuming $\beta>0$ and $g(\beta-0)>0$, we have $\alpha<\infty$ and (3)

$$
\tilde{g}(x)=\alpha g(x \beta) \text { a.e. on }[0,1] \text {. }
$$

Thus, if $\tilde{g}(x)$ has a constant value on $(u, v)$, then $g(x)$ must be constant on the interval $(u \beta, v \beta)$. Furthermore, if $\underline{d}>0$, then for every $g \in G\left(X_{n}\right)$ we have (7)

$$
(\underline{d} / \bar{d}) x \leq g(x) \leq(\bar{d} / \underline{d}) x
$$

for every $x \in[0,1]$. Thus, there exists a sequence $\beta_{k} \in(0,1)$ such that $\beta_{k} \searrow 0$ and $g(x)$ increases in $\beta_{k}, g\left(\beta_{k}\right)>0, k=1,2, \ldots$ For such $\beta_{k}, g(x)$, applying the Helly principle, we can find sequences $\alpha_{k}$ and $\tilde{g}_{k}(x) \in G\left(X_{n}\right)$ such that

$$
\tilde{g}_{k}(x)=\alpha_{k} g\left(x \beta_{k}\right)
$$

a.e. on $[0,1]$. Every $\tilde{g}_{k}(x)$ has a constant value on the interval $(u, v)$, hence, $g(x)$ must be constant on the intervals $\left(u \beta_{k}, v \beta_{k}\right)$ for $k=1,2, \ldots$

\subsection{Transformation of $X_{n}$ by $1 / x \bmod 1$}

The mapping $1 / x \bmod 1$ transforms the block $X_{n}$ to the block

$$
Z_{n}=\left(\frac{x_{n}}{x_{1}}, \frac{x_{n}}{x_{2}}, \ldots, \frac{x_{n}}{x_{n}}\right) \bmod 1 .
$$

For example, the block sequence $X_{n}=\left(\frac{1}{n}, \frac{2}{n}, \ldots, \frac{n}{n}\right), n=1,2, \ldots$ which is u.d. is transformed to the block sequence

$$
Z_{n}=\left(\frac{n}{1}, \frac{n}{2}, \ldots, \frac{n}{n}\right) \bmod 1, \quad n=1,2, \ldots
$$


which has a.d.f.

$$
g(x)=\int_{0}^{1} \frac{1-t^{x}}{1-t} \mathrm{~d} t=\sum_{n=1}^{\infty} \frac{x}{n(n+x)}=\gamma_{0}+\frac{\Gamma^{\prime}(1+x)}{\Gamma(1+x)},
$$

where $\gamma_{0}$ is Euler's constant. This was proved by G. P óly a, (see I. J. S c h o e nberg [17]). The following theorem, which generalizes [12, p. 56, Th. 7.6] describes a relation between $G\left(X_{n}\right)$ and $G\left(Z_{n}\right)$.

Theorem 25 ([9, Th. 7]). If every $g(x) \in G\left(X_{n}\right)$ is continuous on $[0,1]$, then

$$
G\left(Z_{n}\right)=\left\{\tilde{g}(x)=\sum_{n=1}^{\infty} g(1 / n)-g(1 /(n+x)) ; g(x) \in G\left(X_{n}\right)\right\} .
$$

Pr o o f. For $f(x)=1 / x \bmod 1$ we have $f^{-1}([0, t))=\cup_{i=1}^{\infty}(1 /(t+i), 1 / i]$. Thus $F\left(Z_{n}, t\right)=\sum_{i=1}^{\infty}\left(F\left(X_{n}, 1 / i\right)-F\left(X_{n}, 1 /(t+i)\right)\right)$.

$1^{0}$. Assume that $F\left(X_{n_{k}}, x\right) \rightarrow g(x)$, where $g(x)$ is everywhere continuous on $[0,1]$. Thus

$$
\begin{aligned}
\sum_{i=1}^{K}\left(F\left(X_{n_{k}}, 1 / i\right)-F\left(X_{n_{k}}, 1 /(t+i)\right)\right) & \rightarrow \sum_{i=1}^{K}(g(1 / i)-g(1 /(t+i))) \\
\sum_{i=K+1}^{\infty}\left(F\left(X_{n_{k}}, 1 / i\right)-F\left(X_{n_{k}}, 1 /(t+i)\right)\right) & \leq F\left(X_{n_{k}}, 1 /(K+1)\right) \\
& \rightarrow g(1 /(K+1)) \rightarrow 0 .
\end{aligned}
$$

Thus $F\left(Z_{n_{k}}, t\right) \rightarrow \tilde{g}(t)=\sum_{i=1}^{\infty}(g(1 / i)-g(1 /(t+i)))$ for $t \in[0,1]$.

$2^{0}$. Assume that $F\left(Z_{n_{k}}, t\right) \rightarrow \tilde{g}(t)$ weakly. From $n_{k}$ there can be selected $n_{k}^{\prime}$ such that $F\left(X_{n_{k}^{\prime}}, x\right) \rightarrow g(x)$. Assuming continuity of $g(x)$, we apply $1^{0}$.

\section{Examples}

ExAmple 1 (24]). Put $x_{n}=p_{n}$, the $n$th prime and denote

$$
X_{n}=\left(\frac{2}{p_{n}}, \frac{3}{p_{n}}, \ldots, \frac{p_{n-1}}{p_{n}}, \frac{p_{n}}{p_{n}}\right) .
$$

The sequence of blocks $X_{n}$ is u.d. and therefore the ratio sequence $p_{m} / p_{n}$, $m=1,2, \ldots, n, n=1,2, \ldots$ is u.d. in $[0,1]$. This generalizes a result of $\mathrm{A}$. S c h i $\mathrm{n}$ zel (cf. W. S i e r piński $\left(1964\right.$, p. 155)). Note that from u.d. of $X_{n}$ applying for the $L^{2}$ discrepancy of $X_{n}$ we get the following interesting limit

$$
\lim _{n \rightarrow \infty} \frac{1}{n^{2} p_{n}} \sum_{i, j=1}^{n}\left|p_{i}-p_{j}\right|=\frac{1}{3} \text {. }
$$


ExAmple 2 ([24, Ex. 11.1]). Let $\gamma, \delta$, and $a$ be given real numbers satisfying $1 \leq \gamma<\delta \leq a$. Let $x_{n}$ be an increasing sequence of all integer points lying in the intervals

$$
(\gamma, \delta),(\gamma a, \delta a), \ldots,\left(\gamma a^{k}, \delta a^{k}\right), \ldots
$$

Then $G\left(X_{n}\right)=\left\{g_{t}(x) ; t \in[0,1]\right\}$, where $g_{t}(x)$ has constant values

$$
g_{t}(x)=\frac{1}{a^{i}(1+t(a-1))} \quad \text { for } \quad x \in \frac{(\delta, a \gamma)}{a^{i+1}(t \delta+(1-t) \gamma)}, \quad i=0,1,2, \ldots
$$

and on the component intervals it has a constant derivative

$$
\begin{gathered}
g_{t}^{\prime}(x)=\frac{t \delta+(1-t) \gamma}{(\delta-\gamma)\left(\frac{1}{a-1}+t\right)} \quad \text { for } x \in \frac{(\gamma, \delta)}{a^{i+1}(t \delta+(1-t) \gamma)}, \quad i=0,1,2, \ldots \\
\quad \text { and } x \in\left(\frac{\gamma}{t \delta+(1-t) \gamma}, 1\right)
\end{gathered}
$$

where

$$
F\left(X_{n_{k}}, x\right) \rightarrow g_{t}(x) \text { for } n_{k} \quad \text { for which } \quad x_{n_{k}}=\left[a^{k} \gamma+t a^{k}(\delta-\gamma)\right] .
$$

Here we write $(x z, y z)=(x, y) z$ and $(x / z, y / z)=(x, y) / z$. Then the set $G\left(X_{n}\right)$ has the following properties:

$1^{0}$. Every $g \in G\left(X_{n}\right)$ is continuous.

$2^{0}$. Every $g \in G\left(X_{n}\right)$ has infinitely many intervals with constant values, i.e., with $g^{\prime}(x)=0$, and in the infinitely many complement intervals it has a constant derivative $g^{\prime}(x)=c$, where $\frac{1}{\bar{d}} \leq c \leq \underline{1}$ and for lower $\underline{d}$ and upper $\bar{d}$ asymptotic density of $x_{n}$ we have

$$
\underline{d}=\frac{(\delta-\gamma)}{\gamma(a-1)}, \quad \bar{d}=\frac{(\delta-\gamma) a}{\delta(a-1)} .
$$

$3^{0}$. The graph of every $g \in G\left(X_{n}\right)$ lies in the intervals

$$
\left[\frac{1}{a}, 1\right] \times\left[\frac{1}{a}, 1\right] \cup\left[\frac{1}{a^{2}}, \frac{1}{a}\right] \times\left[\frac{1}{a^{2}}, \frac{1}{a}\right] \cup \ldots
$$

Moreover, the graph $g$ in $\left[\frac{1}{a^{k}}, \frac{1}{a^{k-1}}\right] \times\left[\frac{1}{a^{k}}, \frac{1}{a^{k-1}}\right]$ is similar to the graph of $g$ in $\left[\frac{1}{a^{k+1}}, \frac{1}{a^{k}}\right] \times\left[\frac{1}{a^{k+1}}, \frac{1}{a^{k}}\right]$ with coefficient $\frac{1}{a}$. Using the parametric expression, it can be written for all $x \in\left(\frac{1}{a^{i+1}}, \frac{1}{a^{i}}\right)$ that $g_{t}(x)=\frac{g_{t}\left(a^{i} x\right)}{a^{i}}$, $i=0,1,2, \ldots$.

$4^{0}$. $G\left(X_{n}\right)$ is connected and the upper distribution function $\bar{g}(x)=g_{0}(x) \in$ $G\left(X_{n}\right)$ and the lower distribution function $\underline{g}(x) \notin G\left(X_{n}\right)$. The graph of $\underline{g}(x)$ on $\left[\frac{1}{a}, 1\right] \times\left[\frac{1}{a}, 1\right]$ coincides with the graph of

$$
y(x)=\left(1+\frac{1}{\underline{d}}\left(\frac{1}{x}-1\right)\right)^{-1}
$$




\section{DISTRIBUTION FUNCTIONS OF RATIO SEQUENCES. AN EXPOSITORY PAPER}

on $\left[\frac{\gamma}{\delta}, 1\right]$, further, on $\left[\frac{1}{a}, \frac{\gamma}{\delta}\right]$ we have $\underline{g}(x)=\frac{1}{a}$.

$5^{0} . G\left(X_{n}\right)=\left\{\frac{g_{0}(x \beta)}{g_{0}(\beta)} ; \beta \in\left[\frac{1}{a}, \frac{\delta}{a \gamma}\right]\right\}$.

For the proofs of $1^{0} .-5^{0}$. we only note:

Assume that $x_{n} \in a^{k}(\gamma, \delta), i, i+1, i+2, \cdots \in a^{j}(\gamma, \delta)$ for some $j<k$, and let $F\left(X_{n}, x\right) \rightarrow g(x)$ for some sequence of $n$. Then $g(x)$ has a constant derivative in the intervals containing $\frac{i}{x_{n}}, \frac{i+1}{x_{n}}, \frac{i+2}{x_{n}}, \ldots$, since

$$
\frac{\frac{1}{n}}{\frac{i+1}{x_{n}}-\frac{i}{x_{n}}}=\frac{x_{n}}{n},
$$

and thus $\frac{x_{n}}{n}$ must be convergent to $g^{\prime}(x)$, so $\frac{1}{\bar{d}} \leq g^{\prime}(x) \leq \frac{1}{\underline{d}}$. For

$$
x_{n}=\left[t a^{k} \delta+(1-t) a^{k} \gamma\right]
$$

we can find

$$
\begin{aligned}
g^{\prime}(x)=\lim _{n \rightarrow \infty} \frac{x_{n}}{n} & =\lim _{k \rightarrow \infty} \frac{a^{k}(t \delta+(1-t) \gamma)}{\sum_{j=0}^{k-1} a^{j}(\delta-\gamma)+a^{k}(t \delta+(1-t) \gamma)-a^{k} \gamma} \\
& =\frac{t \delta+(1-t) \gamma}{(\delta-\gamma)\left(\frac{1}{a-1}+t\right)} .
\end{aligned}
$$

Using Theorem [18 and [3, Ex. 3] we shall add the following properties moreover:

$6^{0}$. By definition (5) of the local asymptotic density $d_{g}$ and by (53) for $g(x)=$ $g_{t}(x)$ we have

$$
\begin{aligned}
d_{g_{t}}=\lim _{k \rightarrow \infty} \frac{n_{k}}{x_{n_{k}}} & =\lim _{k \rightarrow \infty} \frac{\sum_{i=0}^{k-1} a^{i}(\delta-\gamma)+t a^{k}(\delta-\gamma)}{a^{k} \gamma+t a^{k}(\delta-\gamma)} \\
& =\frac{(\delta-\gamma)(1+t(a-1))}{(a-1)(\gamma+t(\delta-\gamma))}
\end{aligned}
$$

and for $t=0$ we have $d_{g_{0}}=\underline{d}$ and for $t=1$ we have $d_{g_{1}}=\bar{d}$ and we see

$$
g_{t}^{\prime}(x)=\frac{1}{d_{g_{t}}}
$$

for $x$ with the constant derivative of $g_{t}(x)$.

$7^{0}$. For the function $h_{1, g}(x)$ defined in (26), putting $g(x)=g_{t}(x)$, we have:

$$
\begin{gathered}
\frac{\underline{d}}{d_{g_{t}}}=\frac{\gamma+t(\delta-\gamma)}{\gamma(1+t(a-1))}, \frac{1-d_{g_{t}}}{1-\underline{d}}=\frac{\gamma}{\gamma+t(\delta-\gamma)}, \\
\frac{\underline{d}}{d_{g_{t}}} \frac{1-d_{g_{t}}}{1-\underline{d}}=\frac{1}{1+t(a-1)} .
\end{gathered}
$$


Then

$$
h_{1, g_{t}}(x)= \begin{cases}x \frac{\gamma+t(\delta-\gamma)}{\gamma(1+t(a-1))}, & \text { for } x \in\left(0, \frac{\gamma}{\gamma+t(\delta-\gamma)}\right), \\ x \frac{1}{d_{g_{t}}}+1-\frac{1}{d_{g_{t}}}, & \text { for } x \in\left(\frac{\gamma}{\gamma+t(\delta-\gamma)}, 1\right),\end{cases}
$$

see the following figure.

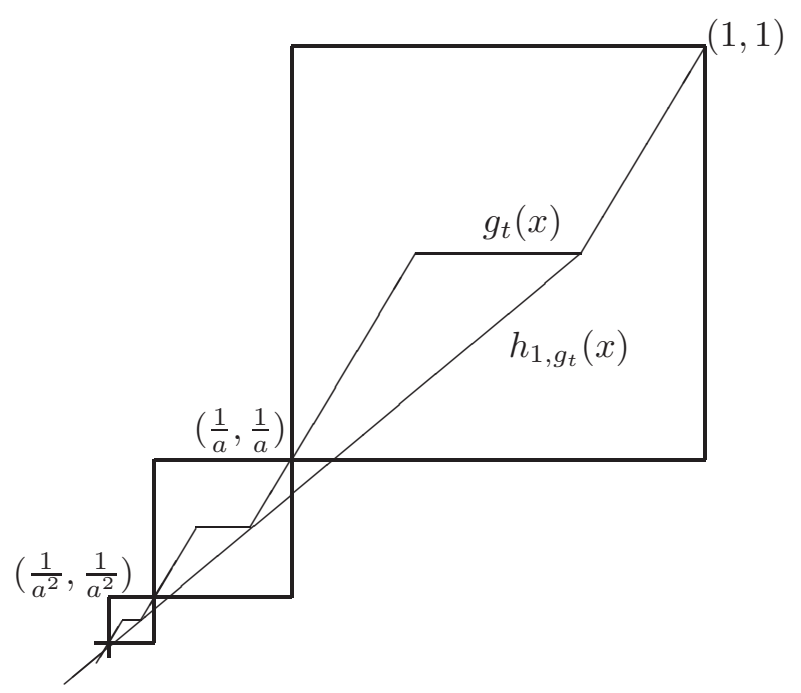

Figure: $g_{t}(x)$ and $h_{1, g_{t}}(x)$.

$8^{0}$. In the proof of the upper bound (29) we have proved that $1-\int_{0}^{1} h_{1, g}(x) \mathrm{d} x$ is maximal for $d_{g}=\min (\sqrt{\underline{d}}, \bar{d})$. Let $t_{0} \in[0,1]$ be such that $d_{g_{t_{0}}}=\min (\sqrt{\underline{d}}, \bar{d})$ and $t_{0}$ can be computed by inverse formula to (54)

$$
t=\frac{d_{g_{t}}(a-1) \gamma-(\delta-\gamma)}{(\delta-\gamma)(a-1)\left(1-d_{g_{t}}\right)}
$$

$9^{0}$. Let $P(t)$ be the area in $\left[\frac{1}{a}, 1\right] \times\left[\frac{1}{a}, 1\right]$ bounded by the graph of $g_{t}(x)$. Then

$$
\begin{aligned}
\int_{0}^{1} g_{t}(x) \mathrm{d} x= & P(t) \frac{1}{1-\frac{1}{a^{2}}}+\frac{1}{a+1} \\
= & \frac{1}{2}+\frac{1}{2} \cdot \frac{1}{(a+1)} \cdot \frac{(\gamma a-\delta)}{(1+t(a-1))(\gamma+t(\delta-\gamma))} \\
& +\frac{1}{2} \cdot \frac{t(\delta-\gamma a)}{(1+t(a-1))(\gamma+t(\delta-\gamma))}
\end{aligned}
$$

and since $g_{0}(x)=\bar{g}(x)$ we have that the $\max _{t \in[0,1]} \int_{0}^{1} g_{t}(x) \mathrm{d} x$ is attained at $t=0$. Using derivative of $P(t)$ it can be see that the $\min _{t \in[0,1]} \int_{0}^{1} g_{t}(x) \mathrm{d} x$ 


\section{DISTRIBUTION FUNCTIONS OF RATIO SEQUENCES. AN EXPOSITORY PAPER}

is attained at $t=1$. It also follows from the fact that for $x_{n+1}=x_{n}+1$ we have

$$
\begin{aligned}
& \frac{1}{n+1} \sum_{i=1}^{n+1} \frac{x_{i}}{x_{n+1}}-\frac{1}{n} \sum_{i=1}^{n} \frac{x_{i}}{x_{n}} \\
& =\frac{1}{n+1}-\left(\frac{1}{x_{n}+1}+\frac{1}{n+1} \cdot \frac{1}{1+\frac{1}{x_{n}}}\right)\left(\frac{1}{n} \sum_{i=1}^{n} \frac{x_{i}}{x_{n}}\right)>0
\end{aligned}
$$

because $c_{1}(x) \notin G\left(X_{n}\right)$ and thus $\lim _{\sup _{n \rightarrow \infty}} \frac{1}{n} \sum_{i=1}^{n} \frac{x_{i}}{x_{n}}<1$. Now, denoting the index $n_{k}$ for $x_{n_{k}}=\left[a^{k} \delta\right]$, the limsup of $\frac{1}{n} \sum_{i=1}^{n} \frac{x_{i}}{x_{n}}$ is attained over $n=n_{k}, k=0,1,2, \ldots$ and for such $n_{k}$ we have $F\left(X_{n_{k}}, x\right) \rightarrow g_{1}(x)$ for $x \in[0,1]$.

$10^{0}$. Thus we have

$$
\begin{aligned}
& \liminf _{n \rightarrow \infty} \frac{1}{n} \sum_{i=1}^{n} \frac{x_{i}}{x_{n}}=1-\int_{0}^{1} g_{0}(x) \mathrm{d} x=\frac{1}{2}-\frac{1}{2} \cdot \frac{1}{(a+1)}\left(\frac{\gamma a-\delta}{\gamma}\right), \\
& \limsup _{n \rightarrow \infty} \frac{1}{n} \sum_{i=1}^{n} \frac{x_{i}}{x_{n}}=1-\int_{0}^{1} g_{1}(x) \mathrm{d} x=\frac{1}{2}+\frac{1}{2} \cdot \frac{1}{(a+1)}\left(\frac{\gamma a-\delta}{\delta}\right) .
\end{aligned}
$$

The upper bound (29) coincides with the maximal value of $1-\int_{0}^{1} h_{1, g}(x) \mathrm{d} x$ attained for $d_{g}=\min (\sqrt{\underline{d}}, \bar{d})$. Since $1-\int_{0}^{1} g_{1}(x) \mathrm{d} x$ is maximal for all $1-\int_{0}^{1} g_{t}(x) \mathrm{d} x, t \in[0,1]$ and $1-\int_{0}^{1} g_{1}(x) \mathrm{d} x \leq 1-\int_{0}^{1} h_{1, g_{1}}(x) \mathrm{d} x$ then the upper bound (60) satisfies (29).

$11^{0}$. Using explicit formulas

$$
\underline{d}=\frac{(\delta-\gamma)}{\gamma(a-1)}, \quad \bar{d}=\frac{(\delta-\gamma) a}{\delta(a-1)}
$$

for asymptotic densities we see again that (59) and (60) satisfy (28) and (29), respectively, in Theorem 19.

ExAmple 3 ( [9, Ex. 2]). Let $x_{n}$ and $y_{n}, n=1,2, \ldots$, be two strictly increasing sequences of positive integers such that for the related block sequences $X_{n}=\left(\frac{x_{1}}{x_{n}}, \ldots, \frac{x_{n}}{x_{n}}\right)$ and $Y_{n}=\left(\frac{y_{1}}{y_{n}}, \ldots, \frac{y_{n}}{y_{n}}\right)$, we have singleton for both $G\left(X_{n}\right)=$ $\left\{g_{1}(x)\right\}$ and $G\left(Y_{n}\right)=\left\{g_{2}(x)\right\}$. Furthermore, let $n_{k}, k=1,2, \ldots$, be an increasing sequence of positive integers such that $N_{k}=\sum_{i=1}^{k} n_{i}$ satisfies $\frac{n_{k}}{N_{k}} \rightarrow 1$. Denote by $z_{n}$ the following increasing sequence of positive integers composed by blocks (here we use the notation $a(b, c, d, \ldots)=(a b, a c, a d, \ldots)$ )

$$
\left(x_{1}, \ldots, x_{n_{1}}\right), x_{n_{1}}\left(y_{1}, \ldots, y_{n_{2}}\right), x_{n_{1}} y_{n_{2}}\left(x_{1}, \ldots, x_{n_{3}}\right), x_{n_{1}} y_{n_{2}} x_{n_{3}}\left(y_{1}, \ldots, y_{n_{4}}\right), \ldots
$$




\section{OTO STRAUCH}

Then the sequence of blocks $Z_{n}=\left(\frac{z_{1}}{z_{n}}, \ldots, \frac{z_{n}}{z_{n}}\right)$ has the set of d.f.s

$$
\begin{aligned}
G\left(Z_{n}\right)=\left\{g_{1}(x), g_{2}(x), c_{0}(x)\right\} & \cup\left\{g_{1}\left(x y_{n}\right) ; n=1,2, \ldots\right\} \\
& \cup\left\{g_{2}\left(x x_{n}\right) ; n=1,2, \ldots\right\} \\
& \cup\left\{\frac{1}{1+\alpha} c_{0}(x)+\frac{\alpha}{1+\alpha} g_{1}(x) ; \alpha \in[0, \infty)\right\}, \\
& \cup\left\{\frac{1}{1+\alpha} c_{0}(x)+\frac{\alpha}{1+\alpha} g_{2}(x) ; \alpha \in[0, \infty)\right\},
\end{aligned}
$$

where $g_{1}\left(x y_{n}\right)=1$ if $x y_{n} \geq 1$, similarly for $g_{2}\left(x x_{n}\right)$.

Pr o of. For every $n=1,2, \ldots$ there exists an integer $k$ such that

$$
N_{k-1}<n \leq N_{k}
$$

(here $N_{0}=0$ ). Put $n^{\prime}=n-N_{k-1}$. For every $n$ we have

$$
z_{n}= \begin{cases}x_{n_{1}} y_{n_{2}} \ldots x_{n_{k-1}} y_{n^{\prime}} & \text { if } k \text { is even } \\ x_{n_{1}} y_{n_{2}} \ldots y_{n_{k-1}} x_{n^{\prime}} & \text { if } k \text { is odd }\end{cases}
$$

Firstly we assume that $k$ is even. Then $Z_{n}$ has the form

$$
\begin{aligned}
& Z_{n}= \\
& \left(\ldots, \frac{x_{n_{1}} y_{n_{2}} \ldots y_{n_{k-2}}\left(x_{1}, \ldots, x_{n_{k-1}}\right)}{x_{n_{1}} y_{n_{2}} \ldots x_{n_{k-1}} y_{n^{\prime}}}, \frac{x_{n_{1}} y_{n_{2}} \ldots x_{n_{k-1}}\left(y_{1}, \ldots, y_{n^{\prime}}\right)}{x_{n_{1}} y_{n_{2}} \ldots x_{n_{k-1}} y_{n^{\prime}}}\right)= \\
& \left(\ldots, \frac{1}{x_{n_{k-1}} y_{n^{\prime}}}\left(\frac{y_{1}}{y_{n_{k-2}}}, \ldots, \frac{y_{n_{k-2}}}{y_{n_{k-2}}}\right), \frac{1}{y_{n^{\prime}}}\left(\frac{x_{1}}{x_{n_{k-1}}}, \ldots, \frac{x_{n_{k-1}}}{x_{n_{k-1}}}\right),\left(\frac{y_{1}}{y_{n^{\prime}}}, \ldots, \frac{y_{n^{\prime}}}{y_{n^{\prime}}}\right)\right)
\end{aligned}
$$

and thus for $x>\frac{1}{x_{n_{k-1}}}$ we have

$$
\begin{aligned}
F\left(Z_{n}, x\right) & =\frac{N_{k-2}+n_{k-1} F\left(X_{n_{k-1}}, x y_{n^{\prime}}\right)+n^{\prime} F\left(Y_{n^{\prime}}, x\right)}{N_{k-1}+n^{\prime}} \\
& =\frac{N_{k-2}}{N_{k-1}+n^{\prime}}+\frac{\frac{n_{k-1}}{N_{k-1}}}{1+\frac{n^{\prime}}{N_{k-1}}} F\left(X_{n_{k-1}}, x y_{n^{\prime}}\right)+\frac{1}{1+\frac{N_{k-1}}{n^{\prime}}} F\left(Y_{n^{\prime}}, x\right) .
\end{aligned}
$$

If $n \rightarrow \infty$, then the first term tends to zero. If $F\left(Z_{n}, x\right) \rightarrow g(x)$ for some sequence of $n$, we can select a subsequence of $n$ 's such that $\frac{n^{\prime}}{N_{k-1}} \rightarrow \alpha$ for some $\alpha \in[0, \infty)$, or $\frac{n^{\prime}}{N_{k-1}} \rightarrow \infty$. For such $n^{\prime}$ we distinguish the following cases: 
(a) If $n^{\prime}=$ constant, then

$\frac{\frac{n_{k-1}}{N_{k-1}}}{1+\frac{n^{\prime}}{N_{k-1}}} F\left(X_{n_{k-1}}, x y_{n^{\prime}}\right) \rightarrow g_{1}\left(x y_{n^{\prime}}\right)\left(\right.$ here $g_{1}\left(x y_{n^{\prime}}\right)=1$ for $\left.x y_{n^{\prime}}>1\right)$

$\frac{1}{1+\frac{N_{k-1}}{n^{\prime}}} F\left(Y_{n^{\prime}}, x\right) \rightarrow 0$

and thus $F\left(Z_{n}, x\right) \rightarrow g_{1}\left(x y_{n^{\prime}}\right)$.

(b) If $n^{\prime} \rightarrow \infty$, then $F\left(X_{n_{k-1}}, x y_{n^{\prime}}\right) \rightarrow 1$; precisely $F\left(X_{n_{k-1}}, x y_{n^{\prime}}\right) \rightarrow c_{0}(x)$.

(b1) If $\frac{n^{\prime}}{N_{k-1}} \rightarrow 0$, then $F\left(Z_{n}, x\right) \rightarrow c_{0}(x)$.

(b2) If $\frac{n^{\prime}}{N_{k-1}} \rightarrow \alpha \in(0, \infty)$, then $F\left(Z_{n}, x\right) \rightarrow \frac{1}{1+\alpha} c_{0}(x)+\frac{\alpha}{1+\alpha} g_{2}(x)$.

(b3) If $\frac{n^{\prime}}{N_{k-1}} \rightarrow \infty$, then $F\left(Z_{n}, x\right) \rightarrow 0+g_{2}(x)$.

For $k$-odd we use a similar computation.

Now, identify $x_{n}=y_{n}$ and select $x_{n}$ such that $g_{1}(x)=x$ (e.g., $x_{n}=n$ or $x_{n}=p_{n}$, the $n$th prime) and put $n_{k}=2^{k^{2}}$ for $k=1,2, \ldots$ Then the set of all d.f.s

$$
\begin{array}{r}
G\left(Z_{n}\right)=\left\{g_{1}(x), c_{0}(x)\right\} \cup\left\{g_{1}\left(x x_{n}\right) ; n=1,2, \ldots\right\} \\
\cup\left\{\frac{1}{1+\alpha} c_{0}(x)+\frac{\alpha}{1+\alpha} g_{1}(x) ; \alpha \in[0, \infty)\right\}
\end{array}
$$

is disconnected, as it can be seen in the figure on the page 174

EXAMPLE 4 . Let $x_{n}, n=1,2, \ldots$, be an increasing sequence of positive integers for which there exists a sequence $n_{k}, k=1,2, \ldots$, of positive integers such that $($ as $k \rightarrow \infty)$

(i) $\frac{n_{k-1}}{n_{k}} \rightarrow 0$,

(ii) $\frac{n_{k}}{x_{n_{k}}} \rightarrow 0$,

(iii) $\frac{x_{n_{k-1}}}{x_{n_{k}}} \rightarrow 0$, and

(iv) $x_{n_{k}-i}=x_{n_{k}}-i$ for $i=0,1, \ldots, n_{k}-n_{k-1}-1$.

Then the sequence of blocks

$$
X_{n}=\left(\frac{x_{1}}{x_{n}}, \frac{x_{2}}{x_{n}}, \ldots, \frac{x_{n}}{x_{n}}\right)
$$

has

$$
G\left(X_{n}\right)=\left\{h_{\alpha}(x) ; \alpha \in[0,1]\right\}
$$




\section{OTO STRAUCH}
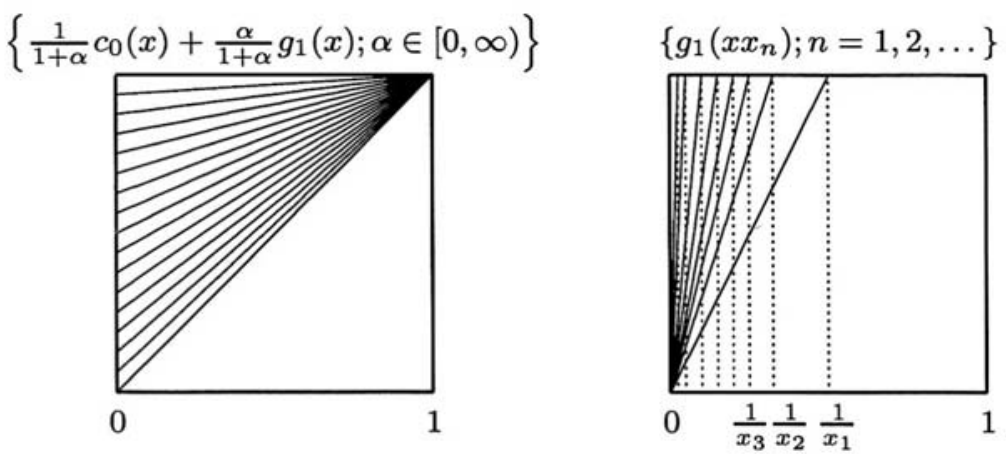

P r o o f. For given $\theta \in[0,1]$ and $n=n_{k}-\left[\theta\left(n_{k}-n_{k-1}\right)\right]$ and by (iv) we have

$$
x_{n}=x_{n_{k}}-\left[\theta\left(n_{k}-n_{k-1}\right)\right] .
$$

For $i \leq n$ we distinguish two cases: $x_{i} \in\left(x_{n_{k-1}}, x_{n}\right]$ and $x_{i} \leq x_{n_{k-1}}$.

(I) For $x_{i} \in\left(x_{n_{k-1}}, x_{n}\right]$ we have

$$
\frac{x_{i}}{x_{n}} \in\left[\frac{x_{n_{k}}-\left(n_{k}-n_{k-1}\right)+1}{x_{n_{k}}-\left[\theta\left(n_{k}-n_{k-1}\right)\right]}, 1\right] \rightarrow[1,1]
$$

as $n \rightarrow \infty$ and for any $\theta \in[0,1]$. The number of such $x_{i}$ 's is

$$
\left(n_{k}-n_{k-1}\right)-\left[\theta\left(n_{k}-n_{k-1}\right)\right]=(1-\theta)\left(n_{k}-n_{k-1}\right)+O(1) .
$$

(II) For $x_{i} \leq x_{n_{k-1}}$ we have

$$
\frac{x_{i}}{x_{n}} \in\left[0, \frac{x_{n_{k-1}}}{x_{n_{k}}-\left[\theta\left(n_{k}-n_{k-1}\right)\right]}\right] \rightarrow[0,0] .
$$

We thus get, for any $x \in(0,1)$ and any sufficiently large $n$,

$$
F\left(X_{n}, x\right)=\frac{n_{k-1}}{n}=\frac{n_{k-1}}{n_{k-1}+(1-\theta)\left(n_{k}-n_{k-1}\right)+O(1)} .
$$

This gives:

(a) If $\theta \leq \varepsilon_{0}<1$, for some fixed $\varepsilon_{0}$, then

$$
F\left(X_{n}, x\right) \rightarrow c_{1}(x) .
$$

(b) If $\theta=1$, then

$$
F\left(X_{n}, x\right) \rightarrow c_{0}(x)
$$

(c) For any $\alpha \in(0,1)$ there exists a sequence $\theta_{k} \rightarrow 1$, as $k \rightarrow \infty$, such that

$$
\frac{n_{k-1}}{n_{k-1}+\left(1-\theta_{k}\right)\left(n_{k}-n_{k-1}\right)} \rightarrow \alpha
$$

and in this case

$$
F\left(X_{n}, x\right) \rightarrow h_{\alpha}(x)
$$




\section{DISTRIBUTION FUNCTIONS OF RATIO SEQUENCES. AN EXPOSITORY PAPER}

Note that the sequences $n_{k}=2^{k^{2}}$ and $x_{n_{k}}=2^{(k+1)^{2}}$ satisfy the assumptions (i), (ii), (iii) and (iv). We also see that $G\left(X_{n}\right)$ is connected but

$$
\begin{aligned}
& F\left(X_{n_{k}+1}, x\right) \rightarrow c_{0}(x), \text { and } \\
& F\left(X_{n_{k}}, x\right) \rightarrow c_{1}(x),
\end{aligned}
$$

a.e. on $[0,1]$ and thus $\rho\left(t_{n_{k}+1}, t_{n_{k}}\right) \rightarrow 1$. Using the permutation $\pi: \mathbb{N} \rightarrow \mathbb{N}$

$$
\begin{aligned}
& 1,2, \ldots, n_{1}, n_{2}, n_{2}-1, n_{2}-2, \ldots, n_{1}+1, n_{2}+1, n_{2}+2, \ldots n_{3}, n_{4}, n_{4}-1, \\
& n_{4}-2, \ldots, n_{3}+1, n_{4}+1, n_{4}+2, \ldots, n_{5}, n_{6}, n_{6}-1, n_{6}-2, \ldots, n_{5}+1, \ldots
\end{aligned}
$$

we have $\rho\left(t_{\pi(n+1)}, t_{\pi(n)}\right) \rightarrow 0$ as $n \rightarrow \infty$, because the "neighbouring" d.f. of $t_{\pi(n)}$ satisfies the scheme

$$
\begin{aligned}
& c_{1}(x), c_{1}(x), \ldots, c_{0}(x), c_{0}(x), \ldots, c_{1}(x), c_{1}(x), \ldots, c_{0}(x), c_{0}(x), \ldots, \\
& c_{1}(x), c_{1}(x), \ldots, c_{0}(x), c_{0}(x), \ldots
\end{aligned}
$$

EXAMPLE 5. In [8] is proved that $\frac{x_{n}}{x_{n+1}} \rightarrow 1$ does not imply that $G\left(X_{n}\right)$ is a singleton. This is a negative answer to the Problem 1.9.2 in [20].

Let $a_{k}, n_{k}, k=1,2, \ldots$, and $x_{n}, n=1,2, \ldots$ be three increasing integer sequences and $h_{1}<h_{2}$ be two positive integers. Assume that

(i) $\frac{n_{k}}{n_{k+1}} \rightarrow 0$ for $k \rightarrow \infty$;

(ii) $\frac{a_{k}}{n_{k+1}} \rightarrow 0$ for $k \rightarrow \infty$;

(iii) for odd $k$ we have

$$
\begin{aligned}
a_{k}^{h_{2}} & \leq x_{n_{k}}=\left(a_{k-1}+n_{k}-n_{k-1}\right)^{h_{1}} \leq\left(a_{k}+1\right)^{h_{2}} \text { and } \\
x_{i} & =\left(a_{k}+i-n_{k}\right)^{h_{2}} \quad \text { for } n_{k}<i \leq n_{k+1} ;
\end{aligned}
$$

(iv) for even $k$ we have

$$
\begin{aligned}
a_{k}^{h_{1}} & \leq x_{n_{k}}=\left(a_{k-1}+n_{k}-n_{k-1}\right)^{h_{2}} \leq\left(a_{k}+1\right)^{h_{1}} \text { and } \\
x_{i} & =\left(a_{k}+i-n_{k}\right)^{h_{1}} \quad \text { for } n_{k}<i \leq n_{k+1} .
\end{aligned}
$$

Then $\frac{x_{n}}{x_{n+1}} \rightarrow 1$ and the set $G\left(X_{n}\right)$ of all distribution functions of the sequence of blocks $X_{n}$ is $G\left(X_{n}\right)=G_{1} \cup G_{2} \cup G_{3} \cup G_{4}$, where

$$
\begin{aligned}
& G_{1}=\left\{x^{\frac{1}{h_{2}}} \cdot t ; t \in[0,1]\right\}, \\
& G_{2}=\left\{x^{\frac{1}{h_{2}}}(1-t)+t ; t \in[0,1]\right\}, \\
& G_{3}=\left\{\max \left(0, x^{\frac{1}{h_{1}}}-\left(1-x^{\frac{1}{h_{1}}}\right) u\right) ; u \in[0, \infty)\right\} \text { and } \\
& G_{4}=\left\{\min \left(1, x^{\frac{1}{h_{1}}} \cdot v\right) ; v \in[1, \infty)\right\} .
\end{aligned}
$$

In [24, Th. 5.2, p. 762$]=$ Theorem [15, it is proved that the condition $\frac{x_{n}}{x_{n+1}} \rightarrow 1$ implies the connectivity of $G\left(X_{n}\right)$ 


\section{OTO STRAUCH}

P r o of. 1. Firstly we prove that for any $h_{1}<h_{2}$ the sequences $a_{k}, n_{k}, x_{n}$ satisfying (i)-(iv) exist:

For $i=1, \ldots, n_{1}$ we put $x_{i}=i^{h_{1}}$ and then we find $a_{1}$ such that $a_{1}^{h_{2}} \leq x_{n_{1}} \leq$ $\left(a_{1}+1\right)^{h_{2}}$. If we have selected, for an odd step $k$, all $a_{i}, i=1,2, \ldots, k-1, x_{i}$, $i=1,2, \ldots, n_{k}$, then we find $a_{k}$ such that $a_{k}^{h_{2}} \leq x_{n_{k}}<\left(a_{k}+1\right)^{h_{2}}$, and then we put $x_{i}=\left(a_{k}+i-n_{k}\right)^{h_{2}}$ for $n_{k}<i \leq n_{k+1}$, where we choose $n_{k+1}$ sufficiently large to satisfy the limits (i) and (ii). For an even step $k$ we proceed similarly replacing $h_{2}$ by $h_{1}$.

2. In contrary to the independence of $a_{k}$ and $n_{k+1}$ we have

$$
\frac{a_{k}}{n_{k}^{\frac{h_{1}}{h_{2}}}} \rightarrow 1 \text { for odd } k \rightarrow \infty, \quad \frac{a_{k}}{n_{k}^{\frac{h_{2}}{h_{1}}}} \rightarrow 1 \text { for even } k \rightarrow \infty .
$$

This follows from (iii) and (iv), directly, e.g., from (iii) we have

$$
\frac{a_{k}^{h_{2}}}{n_{k}^{h_{1}}}<\left(\frac{a_{k-1}}{n_{k}}+1-\frac{n_{k-1}}{n_{k}}\right)^{h_{1}}<\frac{\left(a_{k}+1\right)^{h_{2}}}{n_{k}^{h_{1}}} .
$$

As an application of (62) we have

$$
\frac{a_{k}}{n_{k}} \rightarrow 0 \text { for odd } k \rightarrow \infty, \quad \frac{a_{k}}{n_{k}} \rightarrow \infty \text { for even } k \rightarrow \infty \text {. }
$$

3. Now we prove $\frac{x_{i}}{x_{i+1}} \rightarrow 1$ as $i \rightarrow \infty$. Let $i \in\left(n_{k}, n_{k+1}\right)$ and let, e.g., $k$ be odd. Then by (iii)

$$
\frac{x_{i}}{x_{i+1}}=\left(1-\frac{1}{a_{k}+i+1-n_{k}}\right)^{h_{2}}>\left(1-\frac{1}{a_{k}}\right)^{h_{2}}
$$

and for $i=n_{k}$ again

$$
\frac{x_{n_{k}}}{x_{n_{k}+1}}>\frac{a_{k}^{h_{2}}}{\left(a_{k}+1\right)^{h_{2}}}>\left(1-\frac{1}{a_{k}}\right)^{h_{2}}
$$

which implies the limit 1 as odd $k \rightarrow \infty$. Similarly for even $k$.

4. Let $N \in\left[n_{k}, n_{k+1}\right]$ be an integer sequence (we shall omit the index in $N_{k}$ ) for $k \rightarrow \infty$. For $x \in(0,1)$ we have

$$
\begin{aligned}
F\left(X_{N}, x\right)= & \frac{\#\left\{1 \leq i \leq n_{k-1} ; \frac{x_{i}}{x_{N}}<x\right\}}{N} \\
& +\frac{\#\left\{n_{k-1}<i \leq n_{k} ; \frac{x_{i}}{x_{N}}<x\right\}}{N}+\frac{\#\left\{n_{k}<i \leq N ; \frac{x_{i}}{x_{N}}<x\right\}}{N} \\
= & o(1)+\frac{A}{N}+\frac{B}{N} .
\end{aligned}
$$

To compute $\frac{A}{N}$ for odd $k$ we use

$$
\frac{x_{i}}{x_{N}}=\frac{\left(a_{k-1}+i-n_{k-1}\right)^{h_{1}}}{\left(a_{k}+N-n_{k}\right)^{h_{2}}}<x \Longleftrightarrow i-n_{k-1}<x^{\frac{1}{h_{1}}}\left(a_{k}+N-n_{k}\right)^{\frac{h_{2}}{h_{1}}}-a_{k-1}
$$


and we have

$$
\frac{A}{N}=\frac{\min \left(n_{k}-n_{k-1}, \max \left(0,\left[x^{\frac{1}{h_{1}}}\left(a_{k}+N-n_{k}\right)^{\frac{h_{2}}{h_{1}}}-a_{k-1}\right]\right)\right)}{N} .
$$

Similarly, for even $k$

$$
\frac{A}{N}=\frac{\min \left(n_{k}-n_{k-1}, \max \left(0,\left[x^{\frac{1}{h_{2}}}\left(a_{k}+N-n_{k}\right)^{\frac{h_{1}}{h_{2}}}-a_{k-1}\right]\right)\right)}{N} .
$$

For $\frac{B}{N}$ and odd $k$ we use

$$
\frac{x_{i}}{x_{N}}=\left(\frac{a_{k}+i-n_{k}}{a_{k}+N-n_{k}}\right)^{h_{2}}<x \Longleftrightarrow i-n_{k}<x^{\frac{1}{h_{2}}}\left(a_{k}+N-n_{k}\right)-a_{k}
$$

which gives

$$
\frac{B}{N}=\frac{\min \left(N-n_{k}, \max \left(0,\left[x^{\frac{1}{h_{2}}}\left(a_{k}+N-n_{k}\right)-a_{k}\right]\right)\right)}{N} .
$$

Similarly, for even $k$ we have

$$
\frac{B}{N}=\frac{\min \left(N-n_{k}, \max \left(0,\left[x^{\frac{1}{h_{1}}}\left(a_{k}+N-n_{k}\right)-a_{k}\right]\right)\right)}{N} .
$$

In the following we will distinguish three cases

$$
\frac{n_{k}}{N} \rightarrow t>0, \quad \frac{n_{k}}{N} \rightarrow 0 \text { and } \quad \frac{N}{n_{k+1}} \rightarrow 0, \quad \text { and } \quad \frac{N}{n_{k+1}} \rightarrow t>0 .
$$

5. Now, let $\frac{n_{k}}{N} \rightarrow t>0$ as $k \rightarrow \infty$.

a) Assume that $k$ is odd and compute the limit of $\frac{A}{N}$ by (65). We have $\frac{n_{k}-n_{k-1}}{N} \rightarrow t$ and if $t<1$ we see

$$
x^{\frac{1}{h_{1}}}\left(\frac{a_{k}}{N^{\frac{h_{1}}{h_{2}}}}+\frac{N}{N^{\frac{h_{1}}{h_{2}}}}\left(1-\frac{n_{k}}{N}\right)\right)^{\frac{h_{2}}{h_{1}}}-\frac{a_{k-1}}{N} \rightarrow \infty
$$

since $\frac{N}{N^{\frac{h_{1}}{h_{2}}}}$ for $h_{1}<h_{2}$ is unbounded and by (62)

$$
\frac{a_{k}}{N^{\frac{h_{1}}{h_{2}}}}=\frac{a_{k}}{n_{k}^{\frac{h_{1}}{h_{2}}}}\left(\frac{n_{k}}{N}\right)^{\frac{h_{1}}{h_{2}}} \rightarrow t^{\frac{h_{1}}{h_{2}}}
$$

is bounded. Thus, for $0<t<1$, we have

$$
\frac{A}{N} \rightarrow t \quad \text { for odd } \quad k \rightarrow \infty \text {. }
$$

a1) Let for the moment $t=1$. We have $\frac{a_{k}}{\frac{h_{1}}{n_{2}}} \rightarrow 1$ and 


\section{OTO STRAUCH}

$$
x^{\frac{1}{h_{1}}}\left(\frac{a_{k}}{N^{\frac{h_{1}}{h_{2}}}}+\frac{N-n_{k}}{N^{\frac{h_{1}}{h_{2}}}}\right)^{\frac{h_{2}}{h_{1}}}-\frac{a_{k-1}}{N} \rightarrow x^{\frac{1}{h_{1}}}(1+u)^{\frac{h_{2}}{h_{1}}}
$$

assuming the limit $\frac{N-n_{k}}{N^{\frac{h_{1}}{h_{2}}}} \rightarrow u$, where $u \in[0, \infty)$ can be arbitrary. Put $v=(1+u)^{\frac{h_{2}}{h_{1}}}$. Thus for $t=1$ and corresponding $v \in[1, \infty)$ we have

$$
\frac{A}{N} \rightarrow \min \left(1, x^{\frac{1}{h_{1}}} v\right) \quad \text { for odd } \quad k \rightarrow \infty .
$$

If $\frac{N-n_{k}}{N^{\frac{h_{1}}{h_{2}}}} \rightarrow \infty$, then

$$
\frac{A}{N} \rightarrow 1 \quad \text { for odd } \quad k \rightarrow \infty .
$$

b) Now, again $0<t \leq 1$. For even $k$ in (66) we have

$$
x^{\frac{1}{h_{2}}}\left(\frac{a_{k}}{N^{\frac{h_{2}}{h_{1}}}}+\frac{N}{N^{\frac{h_{2}}{h_{1}}}}\left(1-\frac{n_{k}}{N}\right)\right)^{\frac{h_{1}}{h_{2}}}-\frac{a_{k-1}}{N} \rightarrow x^{\frac{1}{h_{2}}} \cdot t
$$

since by (62)

Thus

$$
\frac{a_{k}}{N^{\frac{h_{2}}{h_{1}}}}=\frac{a_{k}}{n_{k}^{\frac{h_{2}}{h_{1}}}}\left(\frac{n_{k}}{N}\right)^{\frac{h_{2}}{h_{1}}} \rightarrow t^{\frac{h_{2}}{h_{1}}} .
$$

$$
\frac{A}{N} \rightarrow x^{\frac{1}{h_{2}}} \cdot t \quad \text { for even } \quad k \rightarrow \infty .
$$

c) For the limit $\frac{B}{N}$ as odd $k \rightarrow \infty$ we compute (67) by using $\frac{N-n_{k}}{N} \rightarrow 1-t$ and

$$
x^{\frac{1}{h_{2}}}\left(\frac{a_{k}}{N}+1-\frac{n_{k}}{N}\right)-\frac{a_{k}}{N} \rightarrow x^{\frac{1}{h_{2}}}(1-t)
$$

since by (63) we have $\frac{a_{k}}{N}=\frac{a_{k}}{n_{k}} \frac{n_{k}}{N} \rightarrow 0$. Thus

$$
\frac{B}{N} \rightarrow x^{\frac{1}{h_{2}}}(1-t) \quad \text { for odd } \quad k \rightarrow \infty .
$$

d) Again by (63), for even $k$ we have $\frac{a_{k}}{N}=\frac{a_{k}}{n_{k}} \frac{n_{k}}{N} \rightarrow \infty$, then (assuming $x<1$ )

$$
x^{\frac{1}{h_{1}}}\left(\frac{a_{k}}{N}+1-\frac{n_{k}}{N}\right)-\frac{a_{k}}{N} \rightarrow-\infty .
$$

Thus

$$
\frac{B}{N} \rightarrow 0 \quad \text { for even } \quad k \rightarrow \infty .
$$

e) Summing up (69), (172), (73) and (74) we find, for every $x \in(0,1)$,

$$
F\left(X_{N}, x\right) \rightarrow \begin{cases}x^{\frac{1}{h_{2}}}(1-t)+t & \text { for odd } k \rightarrow \infty, \\ x^{\frac{1}{h_{2}}} \cdot t & \text { for even } k \rightarrow \infty\end{cases}
$$


for $\frac{n_{k}}{N} \rightarrow t, 0<t<1$. For $\frac{n_{k}}{N} \rightarrow t=1, \frac{N-n_{k}}{N^{\frac{h_{1}}{h_{2}}}} \rightarrow u$ and $v=(1+u)^{\frac{h_{2}}{h_{1}}}$ we have applying (70)

$$
F\left(X_{N}, x\right) \rightarrow \min \left(1, x^{\frac{1}{h_{1}}} \cdot v\right) \quad \text { for odd } \quad k \rightarrow \infty,
$$

and for $\frac{N-n_{k}}{N^{\frac{h_{1}}{h_{2}}}} \rightarrow \infty$ we have

$$
F\left(X_{N}, x\right) \rightarrow c_{0}(x) \quad \text { for odd } k \rightarrow \infty,
$$

where $c_{0}(x)=1$ for $x \in(0,1)$.

6. In the case $\frac{n_{k}}{N} \rightarrow 0$ and $\frac{N}{n_{k+1}} \rightarrow 0$ we have $\frac{A}{N}=o(1)$ and then it suffices to compute the limit $\frac{B}{N}$ by (67) or (68).

a) Assume that odd $k \rightarrow \infty$. Since $\frac{N-n_{k}}{N} \rightarrow 1$ and by (63) we have $\frac{a_{k}}{N}=$ $\frac{a_{k}}{n_{k}} \frac{n_{k}}{N} \rightarrow 0$ and thus

$$
x^{\frac{1}{h_{2}}}\left(\frac{a_{k}}{N}+1-\frac{n_{k}}{N}\right)-\frac{a_{k}}{N} \rightarrow x^{\frac{1}{h_{2}}} .
$$

b) Assume that even $k \rightarrow \infty$. In this case (by (62) and (ii)) we have

$$
\frac{a_{k}}{N}=\frac{a_{k}}{n_{k}^{\frac{h_{2}}{h_{1}}}} \frac{n_{k}^{\frac{h_{2}}{h_{1}}}}{N}, \quad \frac{a_{k}}{n_{k}^{\frac{h_{2}}{h_{1}}}} \rightarrow 1, \quad \frac{a_{k}}{n_{k+1}} \rightarrow 0, \quad \text { then } \quad \frac{n_{k}^{\frac{h_{2}}{h_{1}}}}{n_{k+1}} \rightarrow 0 .
$$

Thus, for any $u \in[0, \infty)$ we can find a subsequence of $N$ such that

$$
\frac{n_{k}^{\frac{h_{2}}{h_{1}}}}{N} \rightarrow u \text {. }
$$

Then

$$
x^{\frac{1}{h_{1}}}\left(\frac{a_{k}}{N}+1-\frac{n_{k}}{N}\right)-\frac{a_{k}}{N} \rightarrow x^{\frac{1}{h_{1}}}-\left(1-x^{\frac{1}{h_{1}}}\right) u .
$$

c) Summing up (78) and (80) we find for every $x \in(0,1)$

$$
F\left(X_{N}, x\right) \rightarrow \begin{cases}x^{\frac{1}{h_{2}}} & \text { for odd } k \rightarrow \infty, \\ \max \left(0, x^{\frac{1}{h_{1}}}-\left(1-x^{\frac{1}{h_{1}}}\right) u\right) & \text { for even } k \rightarrow \infty\end{cases}
$$

for $\frac{n_{k}}{N} \rightarrow 0, \frac{N}{n_{k+1}} \rightarrow 0$ and for $u \in(0, \infty)$ satisfying (79) if $k$ is even. If $\frac{n_{k}^{\frac{h_{2}}{h_{1}}}}{N} \rightarrow \infty$ then

$$
F\left(X_{N}, x\right) \rightarrow c_{1}(x) \quad \text { for even } \quad k \rightarrow \infty,
$$

where $c_{1}(x)=0$ for $x \in(0,1)$. 


\section{OTO STRAUCH}

7. Finally, let $\frac{N}{n_{k+1}} \rightarrow t>0$. Then $\frac{a_{k}}{N} \rightarrow 0$, because (ii) $\frac{a_{k}}{n_{k+1}} \rightarrow 0$. Computing the limit $\frac{B}{N}$ by (67) or (68) we find

$$
F\left(N_{N}, x\right) \rightarrow \begin{cases}x^{\frac{1}{h_{2}}} & \text { for odd } k \rightarrow \infty, \\ x^{\frac{1}{h_{1}}} & \text { for even } k \rightarrow \infty .\end{cases}
$$

8. Now, assume that $F\left(X_{N}, x\right) \rightarrow g(x)$ for some sequence of $N \in\left[n_{k}, n_{k+1}\right]$, i.e., $g(x) \in G\left(X_{n}\right)$. Then we can find subsequence of $N$ (denoting again as $N$ ) such that $\frac{n_{k}}{N}, \frac{N-n_{k}}{N^{\frac{h_{1}}{h_{2}}}}, \frac{N}{n_{k+1}}$, and $\frac{n_{k}^{\frac{h_{2}}{h_{1}}}}{N}$ converge. Consequently $g(x)$ is contained in the collection of (75), (76), (777), (81), (82) and (83).

Thus the proof is finished.

L. M išík (2004, personal communication) found the following sequence $x_{n}$ for which $c_{1}(x) \in G\left(X_{n}\right)$ and $c_{0}(x) \notin G\left(X_{n}\right)$ and consequently the implication Q.7 in [9] does not hold.

EXAMPLE 6 . Let $x_{n}, n=1,2, \ldots$, be an increasing sequence of positive integers which satisfies the following conditions

(i) if $n_{k}=(k+1)(k-1) ! 2^{\frac{k(k-1)}{2}}$ for $k=1,2, \ldots$, then $x_{n_{k}}=(k+1) n_{k}$,

(ii) if $n_{k}^{\prime}=k(k-2) ! 2^{\frac{k(k-1)}{2}}$ then $x_{n_{k}^{\prime}}=k^{2} n_{k}^{\prime}$,

(iii) if $n=2^{i} n_{k-1}+j, 0 \leq j<2^{i} n_{k-1}$ and $0 \leq i<k-1$ for $k=1,2, \ldots$, then $x_{n}=x_{n_{k-1}}(i+1) 2^{i}+(i+3) k j$ (i.e., $\left.n \in\left[n_{k-1}, n_{k}^{\prime}\right]\right)$,

(iv) if $n \in\left[n_{k}^{\prime}, n_{k}\right]$ for $k=1,2, \ldots$, then $x_{n}=x_{n_{k}^{\prime}}+n-n_{k}^{\prime}$.

Then for the sequence of blocks

$$
X_{n}=\left(\frac{x_{1}}{x_{n}}, \frac{x_{2}}{x_{n}}, \ldots, \frac{x_{n}}{x_{n}}\right)
$$

we have $c_{1}(x) \in G\left(X_{n}\right)$ but $c_{0}(x) \notin G\left(X_{n}\right)$.

P r o of. We start with the following figure:

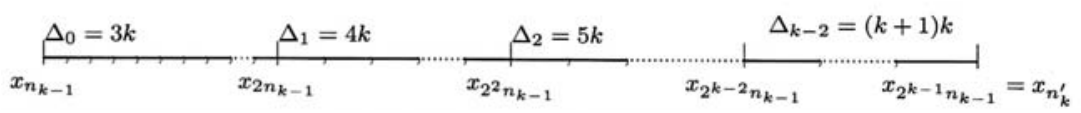

Here for $n$ running through $\left[2^{i} n_{k-1}, 2^{i+1} n_{k-1}\right]$, the $x_{n}$ is equi-distributed in $\left[x_{2^{i} n_{k-1}}, x_{2^{i+1} n_{k-1}}\right]$ with difference $\Delta_{i}$, where $i=0,1, \ldots, k-2$.

5 This and the Theorem 13 imply that $G\left(X_{n}\right) \not \subset\left\{c_{\alpha}(x) ; \alpha \in[0,1]\right\}$. 


\section{DISTRIBUTION FUNCTIONS OF RATIO SEQUENCES. AN EXPOSITORY PAPER}

$1^{0}$. Using the definition of $x_{n}$ we can see that $\frac{x_{n_{k}^{\prime}}}{x_{n_{k}}} \rightarrow 1$ and $\frac{n_{k}^{\prime}}{n_{k}} \rightarrow 0$ and thus we have $c_{1}(x) \in G\left(X_{n}\right)$.

$2^{0}$. On the contrary, assume that there exists increasing sequence $m_{l}^{\prime}<m_{l}$, $l=1,2, \ldots$, such that $m_{l}^{\prime} \in\left[n_{k-1}, n_{k}\right], k=k(l)$, (i) $\frac{x_{m_{l}^{\prime}}}{x_{m_{l}}} \rightarrow 0$ and (ii) $\frac{m_{l}^{\prime}}{m_{l}} \rightarrow 1$ as $l \rightarrow \infty$.

a) If $\left[2^{j} n_{k-1}, 2^{j+1} n_{k-1}\right] \subset\left[m_{l}^{\prime}, m_{l}\right]$ for some $0 \leq j \leq k-2$, then

$$
\frac{m_{l}^{\prime}}{m_{l}} \leq \frac{2^{j} n_{k-1}}{2^{j+1} n_{k-1}}=\frac{1}{2}
$$

which contradicts (ii).

b) If $\left[m_{l}^{\prime}, m_{l}\right] \subset\left[2^{j} n_{k-1}, 2^{j+2} n_{k-1}\right]$, then

$$
\frac{x_{m_{l}^{\prime}}}{x_{m_{l}}} \geq \frac{x_{2^{j} n_{k-1}}}{x_{2^{j+2} n_{k-1}}}=\frac{(j+1) 2^{j}}{(j+3) 2^{j+2}}=\left(1-\frac{2}{j+3}\right) \frac{1}{4}
$$

which contradicts (i).

c) If $\left[n_{k}^{\prime}, n_{k}\right] \subset\left[m_{l}^{\prime}, m_{l}\right]$, then

$$
\frac{m_{l}^{\prime}}{m_{l}} \leq \frac{n_{k}^{\prime}}{n_{k}} \rightarrow 0
$$

which contradicts (ii).

d) If $m_{l}^{\prime} \in\left[2^{k-2} n_{k-1}, n_{k}^{\prime}\right]$ and $m_{l} \in\left[n_{k}^{\prime}, n_{k}\right]$, i.e., $m_{l}=n_{k}^{\prime}+i$, then (because $n_{k}^{\prime}=2^{k-1} m_{k-1}$ and $\left.x_{m_{l}}=x_{n_{k}^{\prime}}+i\right)$

$$
\begin{gathered}
\frac{x_{m_{l}^{\prime}}}{x_{m_{l}}} \geq \frac{x_{2^{k-2} n_{k-1}}}{x_{m_{l}}}=\frac{x_{2^{k-2} n_{k-1}}}{x_{2^{k-1} n_{k-1}}} \cdot \frac{x_{n_{k}^{\prime}}}{x_{m_{l}}}=\left(\frac{k-1}{k}\right) \cdot \frac{1}{2} \cdot \frac{1}{1+\frac{i}{x_{n_{k}^{\prime}}}}, \\
\frac{m_{l}^{\prime}}{m_{l}} \leq \frac{n_{k}^{\prime}}{m_{l}}=\frac{1}{1+\frac{i}{n_{k}^{\prime}}} .
\end{gathered}
$$

Furthermore, (i) implies $\frac{i}{n_{k}^{\prime}} \rightarrow 0$ and (ii) implies $\frac{i}{x_{n_{k}^{\prime}}}=\frac{i}{k^{2} n_{k}^{\prime}} \rightarrow \infty$ which is impossible.

e) If $\left[2 n_{k}, 2^{2} n_{k}\right] \subset\left[m_{l}^{\prime}, m_{l}\right]$ then

$$
\frac{m_{l}^{\prime}}{m_{l}} \leq \frac{2 n_{k}}{2^{2} n_{k}}=\frac{1}{2}
$$

which contradicts (ii).

f) Finally, assume that $m_{l}^{\prime} \in\left[n_{k}^{\prime}, n_{k}\right]$ and $m_{l} \in\left[n_{k}, 2 n_{k}\right]$. Since $x_{2 n_{k}}=4 x_{n_{k}}$, we have

$$
\frac{x_{m_{l}^{\prime}}}{x_{m_{l}}} \geq \frac{x_{n_{k}^{\prime}}}{x_{2 n_{k}}}=\frac{x_{n_{k}^{\prime}}}{4 x_{n_{k}}} \rightarrow \frac{1}{4}
$$

which contradicts (i). 


\section{Historical remarks [21, 1.8.23]}

For every $n=1,2, \ldots$, let

$$
X_{n}=\left(x_{n, 1}, \ldots, x_{n, N_{n}}\right)
$$

be a finite sequence in $[0,1]$. The infinite sequence

$$
\omega=\left(x_{1,1}, \ldots, x_{1, N_{1}}, x_{2,1}, \ldots, x_{2, N_{2}} \ldots\right),
$$

abbreviated as $\omega=\left(X_{n}\right)_{n=1}^{\infty}$, will be called a block sequence associated with the sequence of single blocks $X_{n}, n=1,2, \ldots$ We will distinguish between block sequences and sequences of individual blocks. For the block sequence $\omega=\left(y_{n}\right)_{n=1}^{\infty}$ we can use the step d.f. $F_{N}(x)$ defined as

$$
F_{N}(x)=\frac{\#\left\{n \leq N ; y_{n}<x\right\}}{N}
$$

for $x \in[0,1)$, and $F_{N}(1)=1$. For individual blocks $X_{n}$, we define

$$
F\left(X_{n}, x\right)=\frac{\#\left\{i \leq N_{n} ; x_{n, i}<x\right\}}{N_{n}}
$$

for $x \in[0,1)$ and $F\left(X_{n}, 1\right)=1$.

A d.f. $g$ is a d.f. of the sequence $y_{n}$ if there exists an increasing sequence of positive integers $N_{1}, N_{2}, \ldots$ such that

$$
\lim _{k \rightarrow \infty} F_{N_{k}}(x)=g(x)
$$

a.e. on $[0,1]$.

A d.f. $g$ is a d.f. of the sequence of single blocks $X_{n}$, if there exists an increasing sequence of positive integers $n_{1}, n_{2}, \ldots$ such that

$$
\lim _{k \rightarrow \infty} F\left(X_{n_{k}}, x\right)=g(x)
$$

a.e. on $[0,1]$.

Denote by $G\left(y_{n}\right)$ the set of all d.f. of the sequence $y_{n}$ and denote by $G\left(X_{n}\right)$ the set of all d.f. of the sequence of single blocks $X_{n}$.

In the literature various types of blocks were published:

I. J. S c h o e n berg [17] introduced and studied the asymptotic distribution function (abbreviating a.d.f.) of $X_{n}$ with $N_{n}=n$. For the definition see Section 2 . He gave some criteria and mentioned a result of G. P óly a that

$$
X_{n}=\left(\frac{n}{1}, \frac{n}{2}, \ldots, \frac{n}{n}\right) \bmod 1
$$

has a.d.f. $g(x)=\int_{0}^{1} \frac{1-t^{x}}{1-t} \mathrm{~d} t$. E. H l a w k a in the monograph [10, p. 57-60], called sequences of single blocks $X_{n}$, for $N_{n}=n$, double sequences and, for general $N_{n}$, 


\section{DISTRIBUTION FUNCTIONS OF RATIO SEQUENCES. AN EXPOSITORY PAPER}

$N_{n}$-double sequences. As examples he included a proof of uniform distribution (abbreviating u.d.) for

$$
X_{n}=\left(\frac{1}{n}, \frac{2}{n}, \ldots, \frac{n}{n}\right), \quad \text { and } \quad X_{n}=\left(\frac{1}{n}, \frac{a_{2}}{n}, \ldots, \frac{a_{\phi(n)}}{n}\right),
$$

where $a_{1}=1<a_{2}<\cdots<a_{\phi(n)}$, g.c.d. $\left(a_{i}, n\right)=1$ and $\phi(n)$ denotes Euler's function. U.d. for related block sequences $\omega=\left(X_{n}\right)_{n=1}^{\infty}$ is given in the monograph of L. Kuipers and $\mathrm{H}$. Nied erreiter [12, Lemma 4.1, Example 4.1, p. 136]. G. My ers on [13, p. 172] called a sequence of blocks $X_{n}$ (without any ordering in $X_{n}$ ) a sequence of sets. The same terminology is used by $\mathrm{H}$. Niederreiter in his book 14. Myerson called the associated block sequence $\omega\left(X_{n}\right.$ with some order $)$ an underlying sequence and established criteria for u.d. of $X_{n}$. The sequence of single blocks $X_{n}$ with $N_{n}=n$ is also called a triangular array. R. F. T i c hy [25] gave some examples of u.d. of such $X_{n}$.

Let $x_{n}$ be an increasing sequence of positive integers. Extending a result of S. Knapowski [11], Š. Porubsky, T. Š alát and O. Strauch [15] have investigated a sequence of blocks $X_{n}$ of the type

$$
X_{n}=\left(\frac{1}{x_{n}}, \frac{2}{x_{n}}, \ldots, \frac{x_{n}}{x_{n}}\right) .
$$

They obtained a complete theory for the uniform distribution of the related block sequence $\omega=\left(X_{n}\right)_{n=1}^{\infty}$.

As we see in this paper we have concentrated only on the sequence of blocks $X_{n}, n=1,2, \ldots$, with blocks

$$
X_{n}=\left(\frac{x_{1}}{x_{n}}, \frac{x_{2}}{x_{n}}, \ldots, \frac{x_{n}}{x_{n}}\right) .
$$

Finally, denote by $\mathbb{N}$ the set of all positive integers and if a subset $A \subset \mathbb{N}$ is given, define the ratio set $R(A)$ as $R(A)=\{a / b ; a, b \in A\}$. Main result [22]: For every $A \subset \mathbb{N}$, if the lower asymptotic density $\underline{d}(A) \geq 1 / 2$ then the ratio set $R(A)$ is everywhere dense in $[0, \infty)$. Conversely, if $0 \leq \gamma<1 / 2$ then there exists an $A \subset \mathbb{N}$ such that $\underline{d}(A)=\gamma$ and $R(A)$ is not everywhere dense in $[0, \infty)$.

\section{REFERENCES}

[1] ACZÉL, J.: Lectures on Functional Equations and their Applications. Academic Press, New York, 1966.

[2] BARONE, H. G.: Limit points of sequences and their transforms by methods of summability, Duke Math. J. 5 (1939), 740-752.

[3] BALÁŽ, V.-MIŠÍK, L.-STRAUCH, O.-TÓTH, J. T.: Distribution functions of ratio sequences, III, Publ. Math. Debrecen 82 (2013), 511-529. 


\section{OTO STRAUCH}

[4] BALÁŽ, V.-MIŠÍK, L.-STRAUCH, O.-TÓTH, J.T.: Distribution functions of ratio sequences, IV, Period. Math. Hung. 66 (2013), 1-22.

[5] DRMOTA, M.-TICHY, R. F.: Sequences, Discrepancies and Applications, in: Lect. Notes Math., Vol. 1651, Springer-Verlag, Berlin, 1997.

[6] FILIP, F.-TÓTH, J. T.: On estimations of dispersions of certain dense block sequences, Tatra Mt. Math. Publ. 31 (2005), 65-74.

[7] FILIP, F.-TÓTH, J. T.: Characterization of asymptotic distribution functions of block sequences, 2007 (submitted).

[8] FILIP, F.-MIŠÍK, L.-TÓTH, J. T.: On distribution functions of certain block sequences, Unif. Distrib. Theory 2 (2007), 115-126.

[9] GREKOS, G.-STRAUCH, O.: Distribution functions of ratio sequences, II, Unif. Distrib. Theory 2 (2007), 53-77.

[10] HLAWKA, E.: The theory of uniform distribution, A B Acad. Publ., London, 1984 (English transl. of: Theorie der Gleichverteilung. Bibl. Inst., Mannheim-Wien-Zürich, 1979).

[11] KNAPOWSKI, S.: Über ein Problem der Gleichverteilung, Colloq. Math. 5 (1958), $8-10$.

[12] KUIPERS, L.-NIEDERREITER, H.: Uniform Distribution of Sequences. John Wiley \& Sons, New York 1974, reprint: Dover Publications, Inc. Mineola, New York, 2006.

[13] MYERSON, G.: A sampler of recent developments in the distribution of sequences, in: Number Theory with an Emphasis on the Markoff Spectrum (A.D. Pollington et al., eds.), Provo, UT, 1991, Lect. Notes Pure Appl. Math., Vol. 147, Marcel Dekker, New York, 1993, pp. 163-190.

[14] NIEDERREITER, H.:: Random Number Generation and Quasi-Monte Carlo Methods, SIAM Conf. Ser. Appl. Math., Vol. 63, Society for Industrial and Applied Mathematics, Philadelphia, 1992.

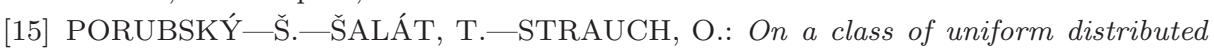
sequences, Math. Slovaca 40 (1990), 143-170.

[16] ŠALÁT, T.: On ratio sets of sets of natural numbers, Acta Arith. XV (1969), $273-278$.

[17] SCHOENBERG, I. J.: Über die asymptotische Vertaeilung reeller Zahlen mod 1, Math. Z. 28 (1928), 171-199.

[18] STRAUCH, O.: A new moment problem of distribution functions in the unit interval, Math. Slovaca 44 (1994), 171-211.

[19] STRAUCH, O.: $L^{2}$ discrepancy, Math. Slovaca 44 (1994), 601-632.

[20] STRAUCH, O.: Unsolved problems, Tatra Mt. Math. Publ. 56 (2013), 109-229, http://udt.mat.savba.sk/

[21] STRAUCH, O.-PORUBSKÝ, Š.: Distribution of Sequences: A Sampler. Peter Lang, Frankfurt am Main, 2005.

[22] STRAUCH, O.-TÓTH, J. T.: Asymptotic density of $A \subset \mathbb{N}$ and density of the ratio set $R(A)$, Acta Arith. LXXXVII (1998), 67-78.

[23] STRAUCH, O.-TÓTH, J. T.: Corrigendum to Theorem 5 of the paper "Asymptotic density of $A \subset \mathbb{N}$ and density of ratio set $R(A)$ " (Acta Arith. 87 (1998), 67-78), Acta Arith. 103.2 (2002), 191-200. 


\section{DISTRIBUTION FUNCTIONS OF RATIO SEQUENCES. AN EXPOSITORY PAPER}

[24] STRAUCH, O.-TÓTH, J. T.: Distribution functions of ratio sequences, Publ. Math. Debrecen 58 (2001), 751-778.

[25] TICHY, R. F.: Three examples of triangular arrays with optimal discrepancy and linear recurrences, in: Applications of Fibonacci Numbers, Vol. 7, Proc. of the 7th Internat. Research Conference on Fibonacci Numbers and Their Appl. (G. E. Bergum et al., eds.), Graz, Austria, 1996, Kluwer Acad. Publ., Dordrecht, 1998, pp. 415-423.

[26] TÓTH, J. T.-MIŠÍK, L.-FILIP, F.: On some properties of dispersion of block sequences of positive integers, Math. Slovaca 54 (2004), 453-464.

Received November 28, 2015

Mathematical Institute

Slovak Academy of Sciences

Stefánikova 49

SK-814-73 Bratislava

SLOVAKIA

E-mail: strauch@mat.savba.sk 